\title{
THE HOMOLOGY OF ITERATED LOOP SPACES
}

\author{
V.A.Smirnov* \\ APPENDIX of F. Sergeraert
}

\section{Introduction}

In recent years, to solve various problems in Algebraic Topology it has been necessary to consider more and more complicated structures on the singular chain complex $C_{*}(X)$ of a topological space $X$ and its homology $H_{*}(X)$.

One of the most difficult problem is the problem of calculating the homology groups of iterated loop spaces. The first steps toward solving this problem were made by J.F.Adams, [1]. To calculate the homology $H_{*}(\Omega X)$ of the loop space $\Omega X$ of a topological space $X$ he introduced the notion of the cobar construction $F K$ on a coalgebra $K$.

Recall that a chain complex $K$ is called a coalgebra if there is given a chain mapping $\nabla: K \rightarrow K \otimes K$ satisfying the associativity relation

$$
(\nabla \otimes 1) \nabla=(1 \otimes \nabla) \nabla
$$

The cobar construction $F K$ of a coalgebra $K$ is a differential algebra that coincides, as a graded algebra, with the tensor algebra $T S^{-1} K$ on the desuspension $S^{-1} K$ of $K$. The generators in $F K$ are denoted by $\left[x_{1}, \ldots, x_{n}\right]$ where $x_{i} \in K$, $1 \leq i \leq n$, and have dimensions

$$
\operatorname{dim}\left[x_{1}, \ldots, x_{n}\right]=\sum_{i=1}^{n} \operatorname{dim}\left(x_{i}\right)-n
$$

The product $\pi: F K \otimes F K \rightarrow F K$ is defined by the formula

$$
\pi\left(\left[x_{1}, \ldots, x_{n}\right] \otimes\left[x_{n+1}, \ldots, x_{n+m}\right]\right)=\left[x_{1}, \ldots, x_{n+m}\right] .
$$

The differential on the generators $[x] \in F K$ is defined by the formula

$$
d[x]=-[d(x)]+\sum(-1)^{\epsilon}\left[x^{\prime}, x^{\prime \prime}\right]
$$

where $\nabla(x)=\sum x^{\prime} \otimes x^{\prime \prime}, \epsilon=\operatorname{dim}\left(x^{\prime}\right)$. On the other elements, the differential is defined as a graded derivation.

The chain complex $C_{*}(X)$ of a topological space $X$ possesses a natural coalgebra structure. Therefore there is defined the cobar construction $F C_{*}(X)$.

J.F.Adams proved that for a simply-connected topological space $X$ there is a chain equivalence of differential algebras

$$
C_{*}(\Omega X) \simeq F C_{*}(X)
$$

In particular, if the topological space $X$ is the suspension of a space $Y$, i.e. $X=S Y$, then the coalgebra structure on $C_{*}(X)$ is can be taken as trivial. Hence 
the chain complex $C_{*}(\Omega X)$ of the loop space $\Omega X$ will be chain equivalent to the tensor algebra $T C_{*}(Y)$ on the chain complex $C_{*}(Y)$.

Unfortunately, the cobar construction $F K$ of a coalgebra $K$ does not admit iteration, because passing to the cobar construction we lose a coalgebra structure. There is, in general, no natural coalgebra structure on the Adams cobar construction $F K$ on a coalgebra $K$.

However H.J.Baues [2] introduced a coalgebra structure on the Adams cobar construction $F C_{*}(X)$ on the chain complex $C_{*}(X)$ of a topological space $X$. This structure was determined using a family of operations

$$
\nabla_{n, m}: C_{*}(X) \rightarrow C_{*}(X)^{\otimes n} \otimes C_{*}(X)^{\otimes m}
$$

of dimensions $n+m-1$. Thus he defined the double cobar construction $F^{2} C_{*}(X)$ and proved that for a 2 -connected topological space $X$ there is a chain equivalence

$$
C_{*}\left(\Omega^{2} X\right) \simeq F^{2} C_{*}(X)
$$

But there is no known appropriate coalgebra structure on the double cobar construction and therefore further iterations are not possible.

In [3] J.P.May introduced the notion of an operad and investigated the structure on iterated loop spaces. This structure is used in [4] to calculate the homology $H_{*}\left(\Omega^{n} S^{n} X\right)$. The homology $H_{*}\left(\Omega^{n} S^{n} X\right)$ has also been investigated in [5], [6] and others.

In [7], [8], [9] the operad methods were transfered from the category of topological spaces to the category of chain complexes. It was shown that on the singular chain complex $C_{*}(X)$ of a topological space $X$ there is a natural $E_{\infty}$-coalgebra structure.

Using this structure, the chain complex $C_{*}\left(\Omega^{n} X\right)$ of the $n$-fold loop space $\Omega^{n} X$ of an $n$-connected topological space $X$ was expressed in terms of the chain complex $C_{*}(X)$ of the space $X$.

Our aim here is to construct a spectral sequence for the homology of iterated loop spaces and produce some calculations.

To do it, we use the $E_{\infty}$-coalgebra structure on the singular chain complex $C_{*}(X)$ of a topological space $X$ (Theorem 1 ). Then we express the chain complex $C_{*}\left(\Omega^{n} X\right)$ of the iterated loop space $\Omega^{n} X$ in terms of the cobar construction of $C_{*}(X)$ (Theorem 2).

After that we consider the spectral sequence of the cobar construction and calculate its first term with $\mathbb{Z} / 2$-coefficients (Theorem 3 ), with $\mathbb{Z} / p$-coefficients (Theorem 3 ') and over a field of characteristic zero (Theorem 3"). Also we give the expression of the $E^{1}$-term as the free $n$-Poisson algebra generated by the homology of $X$ (Theorems 4, 4').

In the cases of $\mathbb{Z} / 2, \mathbb{Z} / p$ and characteristic zero coefficients, we calculate the differential $d^{1}$ on the first term and obtain the expression of the $E^{2}$-term of the spectral sequence (Theorems 5,5 ', 5”).

Finally we apply these results to calculate the homology of the iterated loop spaces of the stunted real and complex projective spaces (Theorem 6, 7, 8), which play important roles in Algebraic Topology. Some calculations with these spaces have been produced by F.Cohen and R. Levi [10].

Note that a general method for calculating the homology of iterated loop spaces 
space of a simplicial set $X$ and its iteration $G^{n} X$. But this construction is very complicated and direct calculations may be produced only using computer methods $[11]$.

The computer calculations may be produced for any topological space, but only in low dimensions. Conversely the spectral sequence calculations may be produced for "nice" spaces in any dimensions. So the computer calculations complement the spectral sequence calculations.

The problem of comparing the computer and the spectral sequence calculations for these spaces was stated by F.Sergeraert. It seems to be very useful for both sides of these calculations.

\section{$\S 1$. Operads and algebras over operads}

Consider the category of chain complexes over a ring $R$. By a symmetric family $\mathcal{E}$ in this category is meant a family $\mathcal{E}=\{\mathcal{E}(j)\}_{j \geq 1}$ of chain complexes $\mathcal{E}(j)$ operated on by the symmetric groups $\Sigma_{j}$.

Given two symmetric families $\mathcal{E}, \mathcal{E}^{\prime}$ we define the symmetric family $\mathcal{E} \otimes \mathcal{E}^{\prime}$ by putting

$$
\left(\mathcal{E} \otimes \mathcal{E}^{\prime}\right)(j)=\mathcal{E}(j) \otimes \mathcal{E}^{\prime}(j)
$$

and $\left(\mathcal{E} \times \mathcal{E}^{\prime}\right)$ by putting $\left(\mathcal{E} \times \mathcal{E}^{\prime}\right)(j)$ equal to the quotient module of the $\Sigma_{j}$-free module generated by the module

$$
\sum_{j_{1}+\cdots+j_{k}=j} \mathcal{E}(k) \otimes \mathcal{E}^{\prime}\left(j_{1}\right) \otimes \cdots \otimes \mathcal{E}^{\prime}\left(j_{k}\right)
$$

modulo the equivalence generated by the relations

$$
\begin{gathered}
x \sigma \otimes x_{1}^{\prime} \otimes \cdots \otimes x_{k}^{\prime} \sim x^{\prime} \otimes x_{\sigma^{-1}(1)}^{\prime} \otimes \cdots \otimes x_{\sigma^{-1}(k)}^{\prime} \cdot \sigma\left(j_{1}, \ldots, j_{k}\right), \\
x \otimes x_{1}^{\prime} \sigma_{1} \otimes \cdots \otimes x_{k}^{\prime} \sigma_{k} \sim x \otimes x_{1}^{\prime} \otimes \cdots \otimes x_{k}^{\prime} \cdot \sigma_{1} \times \cdots \times \sigma_{k} .
\end{gathered}
$$

Here $\sigma\left(j_{1}, \ldots, j_{k}\right)$ is the permutation of a set of $j$ elements obtained by partitioning the set into $k$ blocks of $j_{1}, \ldots, j_{k}$ elements, respectively, and carrying out on these blocks the permutation $\sigma$, while $\sigma_{1} \times \cdots \times \sigma_{k}$ means the image of the element $\left(\sigma_{1}, \ldots, \sigma_{k}\right)$ under the imbedding $\Sigma_{j_{1}} \times \cdots \times \Sigma_{j_{k}} \rightarrow \Sigma_{j}$.

It is easy to see that for symmetric families $\mathcal{E}, \mathcal{E}^{\prime}, \mathcal{F}, \mathcal{F}^{\prime}$, there is the interchange mapping

$$
T:\left(\mathcal{E} \otimes \mathcal{E}^{\prime}\right) \times\left(\mathcal{F} \otimes \mathcal{E}^{\prime}\right) \rightarrow(\mathcal{E} \times \mathcal{F}) \otimes\left(\mathcal{E}^{\prime} \times \mathcal{F}^{\prime}\right)
$$

A symmetric family $\mathcal{E}$ is called an operad if there is given a symmetric-family mapping $\gamma: \mathcal{E} \times \mathcal{E} \rightarrow \mathcal{E}$ such that $\gamma(\gamma \times 1)=\gamma(1 \times \gamma)$ or, what is the same, the following diagram is commutative

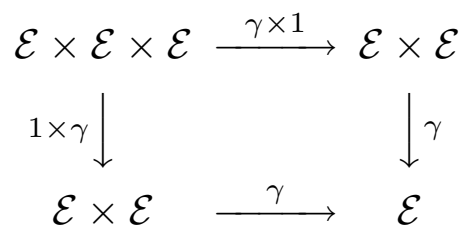

If there exist an element $1 \in \mathcal{E}(1)$ such that $\gamma(1 \otimes x)=\gamma\left(x \otimes 1^{\otimes k}\right)=x$ for all 
A mapping of operads $f: \mathcal{E} \rightarrow \mathcal{E}^{\prime}$ is a mapping of symmetric families for which the following diagram is commutative

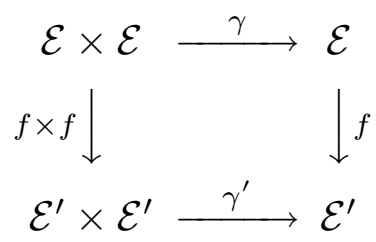

If $\mathcal{E}$ and $\mathcal{E}^{\prime}$ are operads with identities 1 and $1^{\prime}$ respectively, then it is required that $f(1)=1^{\prime}$.

We shall say that an operad $\mathcal{E}$ acts on a symmetric family $\mathcal{F}$ on the left (right) if there is given a mapping $\mu^{\prime}: \mathcal{E} \times \mathcal{F} \rightarrow \mathcal{F}\left(\mu^{\prime \prime}: \mathcal{F} \times \mathcal{E} \rightarrow \mathcal{F}\right)$ such that

$$
\mu^{\prime}(\gamma \times 1)=\mu^{\prime}\left(1 \times \mu^{\prime}\right) \quad\left(\mu^{\prime \prime}(1 \times \gamma)=\mu^{\prime \prime}\left(\mu^{\prime \prime} \times 1\right)\right)
$$

or, what is the same, the following diagrams are commutative

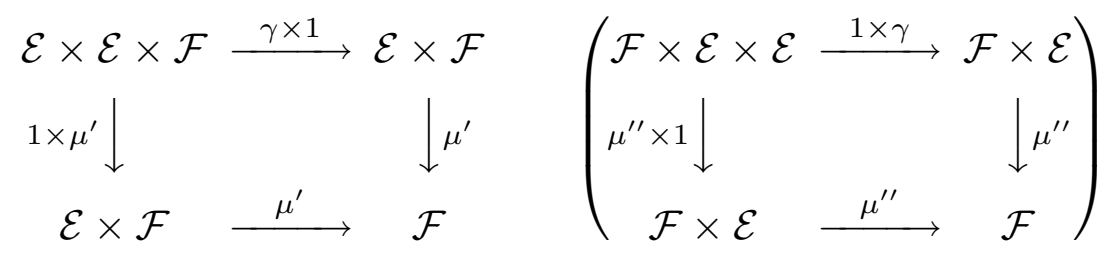

For any chain complex $X$ and symmetric family $\mathcal{E}$, we define chain complexes $\mathcal{E}(X), \overline{\mathcal{E}}(X)$ by putting

$$
\mathcal{E}(X)=\sum_{k} \mathcal{E}(k) \otimes_{\Sigma_{k}} X^{\otimes k}, \quad \overline{\mathcal{E}}(X)=\prod_{k} \operatorname{Hom}_{\Sigma_{k}}\left(\mathcal{E}(k) ; X^{\otimes k}\right) .
$$

If $\mathcal{E}$ is an operad, then the operad structure in $\mathcal{E}$ determines a mapping

$$
\gamma: \mathcal{E}^{2}(X)=\mathcal{E}(\mathcal{E}(X)) \rightarrow \mathcal{E}(X)
$$

such that the correspondence $X \longmapsto \mathcal{E}(X)$ is a monad (also known as a triple) in the category of chain complexes.

Dually, the operad structure in $\mathcal{E}$ determines a mapping

$$
\bar{\gamma}: \overline{\mathcal{E}}(X) \rightarrow \overline{\mathcal{E}}^{2}(X)=\overline{\mathcal{E}}(\overline{\mathcal{E}}(X))
$$

such that the correspondence $X \longmapsto \overline{\mathcal{E}}(X)$ is a comonad in the category of chain complexes.

A chain complex $X$ is called an algebra over the operad $\mathcal{E}$, or simply an $\mathcal{E}$-agebra, if there is given a mapping $\mu: \mathcal{E}(X) \rightarrow X$ satisfying the associativity relation:

$$
\mu \circ \gamma(X)=\mu \circ \mathcal{E}(\mu)
$$

or, what is the same, the following diagram is commutative

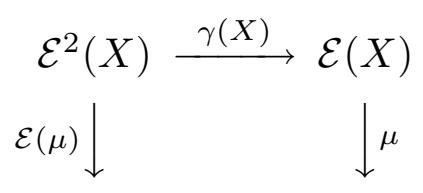


Dually, a chain complex $X$ is called a coalgebra over the operad $\mathcal{E}$, or simply an $\mathcal{E}$-coalgebra, if there is given a mapping $\tau: X \rightarrow \overline{\mathcal{E}}(X)$ satisfying the associativity relation:

$$
\bar{\gamma}(X) \circ \tau=\overline{\mathcal{E}}(\tau) \circ \tau
$$

or, what is the same, the following diagram is commutative

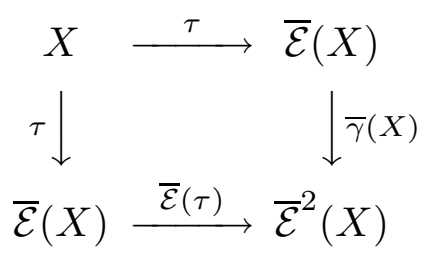

Consider some examples of operads and algebras over operads.

1. The operad $E_{0}=\left\{E_{0}(j)\right\}$, where $E_{0}(j)$ - the free $R$-module with one zero dimensional generator $e(j)$ and trivial action of the symmetric group $\Sigma_{j}$. So $E_{0}(j) \cong$ $R$. The operation $\gamma: E_{0} \times E_{0} \rightarrow E_{0}$ is given by the formula

$$
\gamma\left(e(k) \otimes e\left(j_{1}\right) \otimes \cdots \otimes e\left(j_{k}\right)\right)=e\left(j_{1}+\cdots+j_{k}\right)
$$

It is easy to see that the required relations are satisfied and algebras (coalgebras) over the operad $E_{0}$ are simply commutative and associative algebras (coalgebras).

2. The operad $A=\{A(j)\}$, where $A(j)$ is the $\Sigma_{j}$-free module with one zero dimensional generator $a(j)$. So $A(j) \cong R\left(\Sigma_{j}\right)$. The operation $\gamma: A \times A \rightarrow A$ is given by the formula

$$
\gamma\left(a(k) \otimes a\left(j_{1}\right) \otimes \cdots \otimes a\left(j_{k}\right)\right)=a\left(j_{1}+\cdots+j_{k}\right) .
$$

It is easy to see that the required relations are satisfied and that algebras (coalgebras) over the operad $A$ are simply associative algebras (coalgebras).

3. For a symmetric family $\mathcal{E}$, define the suspension $S \mathcal{E}$ by putting $(S \mathcal{E})(j)=$ $S^{j-1} \mathcal{E}(j)$, the $(j-1)$-fold suspension over $\mathcal{E}(j)$. It is clear that if $\mathcal{E}$ is an operad then the suspension $S \mathcal{E}$ will also be an operad, and if $X$ is an algebra (coalgebra) over an operad $\mathcal{E}$ then the suspension $S X$ will also be an algebra (coalgebra) over the operad $S \mathcal{E}$.

4. For operads $\mathcal{E}, \mathcal{E}^{\prime}$, the tensor product $\mathcal{E} \otimes \mathcal{E}^{\prime}$ evidently is an operad.

5. Let $L$ be the suboperad of the operad $A$ generated by the element

$$
b(2)=a(2)-a(2) T, \quad T \in \Sigma_{2} .
$$

Then algebras (coalgebras) over the operad $L$ will be simply Lie algebras (coalgebras).

Similary, let $L_{n}$ be the suboperad of the operad $S^{n} A$ generated by the element

$$
b_{n}(2)=s^{n} a(2)+(-1)^{n} s^{n} a(2) T .
$$

Then algebras (coalgebras) over the operad $L_{n}$ will be simply $n$-Lie algebras (coalgebras) [4]. It means there is given a Lie bracket of dimension $n$, called an $n$-Lie bracket satisfying the relations

$$
[x, y]+(-1)^{\epsilon}[y, x]=0
$$


where $\epsilon=(\operatorname{dim}(x)+n) \cdot(\operatorname{dim}(y)+n)$. So this is defferent from $N$-Lie meaning $N$-ary bracket [12].

6. Let $P_{n}=E_{0} \times L_{n}$ and the operad structure $\gamma$ is determined by the corresponding structures in $E_{0}, L_{n}$ and by the formulas

$$
\gamma\left(b_{n}(2) \otimes 1 \otimes e(2)\right)=e(2) \otimes b_{n}(2) \otimes 1+e(2) \otimes 1 \otimes b_{n}(2) \cdot(213) .
$$

The operad $P_{n}$ is called the $n$-Poisson operad. Algebras (coalgebras) over this operad called $n$-Poisson algebras (coalgebras). They are commutative algebras together with a Lie bracket of dimension $n$ satisfying the Poisson relation

$$
[x, y \cdot z]=[x, y] z+(-1)^{\delta} y[x, z], \quad[4]
$$

where $\delta=(\operatorname{dim}(x)+n) \cdot \operatorname{dim}(y)$.

7. For any chain complex $X$ define the operads $\mathcal{E}_{X}, \mathcal{E}^{X}$, by putting

$$
\mathcal{E}_{X}(j)=\operatorname{Hom}\left(X^{\otimes j} ; X\right) ; \quad \mathcal{E}^{X}(j)=\operatorname{Hom}\left(X ; X^{\otimes j}\right) .
$$

The actions of the symmetric groups are determined by the permutations of factors of $X^{\otimes j}$ and the operad structures are defined by the formulas

$$
\begin{aligned}
\gamma_{X}\left(f \otimes g_{1} \otimes \cdots \otimes g_{k}\right) & =f \circ\left(g_{1} \otimes \cdots \otimes g_{k}\right), \quad f \in \mathcal{E}_{X}(k), g_{i} \in \mathcal{E}_{X}\left(j_{i}\right) \\
\gamma^{X}\left(f \otimes g_{1} \otimes \cdots \otimes g_{k}\right) & =\left(g_{1} \otimes \cdots \otimes g_{k}\right) \circ f, \quad f \in \mathcal{E}^{X}(k), g_{i} \in \mathcal{E}^{X}\left(j_{i}\right) .
\end{aligned}
$$

Directly from the definitions, it follows that a chain complex $X$ is an algebra (coalgebra) over the operad $\mathcal{E}$ if and only if there is given an operad mapping $\xi: \mathcal{E} \rightarrow \mathcal{E}_{X}\left(\xi: \mathcal{E} \rightarrow \mathcal{E}^{X}\right)$.

Analogously, for chain complexes $X$ and $Y$ there are defined the symmetric families $\mathcal{F}_{X, Y}, \mathcal{F}^{X, Y}$

$$
\mathcal{F}_{X, Y}(j)=\operatorname{Hom}\left(X^{\otimes j} ; Y\right), \quad \mathcal{F}^{X, Y}(j)=\operatorname{Hom}\left(X ; Y^{\otimes j}\right)
$$

and actions

$$
\begin{aligned}
\mu^{\prime}: \mathcal{E}_{Y} \times \mathcal{F}_{X, Y} & \rightarrow \mathcal{F}_{X, Y}, \mu^{\prime \prime}: \mathcal{F}_{X, Y} \times \mathcal{E}_{X} \rightarrow \mathcal{F}_{X, Y} \\
\mu^{\prime}: \mathcal{E}^{X} \times \mathcal{F}^{X, Y} & \rightarrow \mathcal{F}^{X, Y}, \mu^{\prime \prime}: \mathcal{F}^{X, Y} \times \mathcal{E}^{Y} \rightarrow \mathcal{F}^{X, Y}
\end{aligned}
$$

8. One of the most important topological operad is the J.M.Boardman and R.M.Vogt's "little $n$-cubes" operad $E_{n},[13]$.

Let $J$ denote the open interval $(0,1)$ and $J^{n}$ the open $n$-dimensional cube. By an $n$-dimensional little cube is meant an affine embedding $f: J^{n} \rightarrow J^{n}$ with parallel axes. Then $E_{n}(j)$ is the set of ordered $j$-tuples $\left(f^{1}, \ldots, f^{j}\right)$ of $n$-dimensional little cubes $f^{i}: J^{n} \rightarrow J^{n}$ such that images don't intersect. This operad acts on the $n$-fold loop space $\Omega^{n} X$ of a space $X$.

The direct limit of the operads $E_{n}$ over the inclusions $E_{n} \subset E_{n+1}$ is denoted $E_{\infty}$. It is an acyclic operad with free actions of the symmetric groups.

9. It is easy to see that if $\mathcal{E}$ is a topological operad, then its singular chain complex $C_{*}(\mathcal{E})$ is an operad in the category of chain complexes, and if $\mathcal{E}$ acts on a 
Similary, the homology $H_{*}(\mathcal{E})$ of a topological operad $\mathcal{E}$ is an operad in the category of graded modules, and if $\mathcal{E}$ acts on a space $X$, then $H_{*}(\mathcal{E})$ acts on the homology $H_{*}(X)$.

In particular, the homology $H_{*}\left(E_{n}\right)$ of the topological $n$-cubes operad $E_{n}$ is isomorphic to the $n$-Poisson operad $P_{n},[15]$.

So the homology $H_{*}\left(\Omega^{n} X\right)$ of the $n$-fold loop space $\Omega^{n} X, n>1$ is an algebra over the $n$-Poisson operad $P_{n}$.

The operad $C_{*}\left(E_{\infty}\right)$ gives us an example of an acyclic operad in the category of chain complexes with free actions of the symmetric groups.

Note that all acyclic operads with the free actions of the symmetric groups consist of $\Sigma_{j}$-homotopy equivalent chain complexes. We will call such operads: $E_{\infty}$-operads.

10. Another example of an $E_{\infty}$-operad is given simplicial resolutions of the symmetric groups. Denote by $E \Sigma_{*}(j)$ the free simplicial resolution of the symmetric group $\Sigma_{j}$, i.e.

$$
E \Sigma_{*}(j): \Sigma_{j} \leftarrow \Sigma_{j} \times \Sigma_{j} \leftarrow \ldots
$$

The mappings $\gamma: \Sigma_{k} \times \Sigma_{j_{1}} \times \cdots \times \Sigma_{j_{k}} \rightarrow \Sigma_{j_{1}+\cdots+j_{k}}$ induce the operad structure

$$
\gamma_{*}: E \Sigma_{*}(k) \times E \Sigma_{*}\left(j_{1}\right) \times \cdots \times E \Sigma_{*}\left(j_{k}\right) \rightarrow E \Sigma_{*}\left(j_{1}+\cdots+j_{k}\right) .
$$

So $E \Sigma_{*}$ will be an acyclic operad with free actions of the symmetric groups in the category of simplicial sets.

Taking the chain complex $C_{*}\left(E \Sigma_{*}\right)$, we obtain an $E_{\infty}$-operad in the category of chain complexes. Denote it simply by $E \Sigma$.

Note that for any chain operad $\mathcal{E}$, the operad $\mathcal{E} \otimes E \Sigma$ has the same homology and free actions of the symmetric groups. The projection $E \Sigma \rightarrow E_{0}$ induces the projection $\mathcal{E} \otimes E \Sigma \rightarrow \mathcal{E}$. So $\mathcal{E} \otimes E \Sigma$ may be considered as a $\Sigma$-free resolution of the operad $\mathcal{E}$. If $\mathcal{E}$ is an acyclic operad, then $\mathcal{E} \otimes E \Sigma$ will be an $E_{\infty}$-operad.

\section{$\S 2$. On the chain complex of a topological space}

Here we consider structure on the singular chain complex $C_{*}(X)$ of a topological space $X$, and dually on the singular cochain complex $C^{*}(X)$.

Besides the coalgebra structure

$$
\nabla: C_{*}(X) \rightarrow C_{*}(X) \otimes C_{*}(X)
$$

on the chain complex of a topological space, there are coproducts

$$
\nabla_{i}: C_{*}(X) \rightarrow C_{*}(X) \otimes C_{*}(X)
$$

increasing dimensions by $i$ and such that

$$
d\left(\nabla_{i}\right)=\nabla_{i-1}+(-1)^{i} T \nabla_{i-1},
$$

where $T: C_{*}(X) \otimes C_{*}(X) \rightarrow C_{*}(X) \otimes C_{*}(X)$ permutes factors.

Dually, on the cochain complex $C^{*}(X)$ besides the algebra structure 
there are products

$$
\cup_{i}: C^{*}(X) \otimes C^{*}(X) \rightarrow C^{*}(X)
$$

such that $d\left(\cup_{i}\right)=\cup_{i-1}+(-1)^{i} \cup_{i-1} T$.

To describe all operations on the singular chain complex $C_{*}(X)$ of a toplogical space $X$ and on its dual cochain complex $C^{*}(X)$, we consider the corresponding operad.

For $n \geq 0$ denote by $\Delta^{n}$ the normalized chain complex of the standard $n$ dimensional simplex. Then $\Delta^{*}=\left\{\Delta^{n}\right\}$ is a cosimplicial object in the category of chain complexes. Consider also the cosimplicial object $\left(\Delta^{*}\right)^{\otimes j}=\Delta^{*} \otimes \cdots \otimes \Delta^{*}$ and

$$
E^{\Delta}(j)=\operatorname{Hom}\left(\Delta^{*} ;\left(\Delta^{*}\right)^{\otimes j}\right),
$$

where Hom is considered in the category of cosimplicial objects.

The family $E^{\Delta}=\left\{E^{\Delta}(j)\right\}$ will be the operad for which the actions of the symmetric groups and the operad structure are defined similary to the corresponding structures for the above defined operad $\mathcal{E}^{X}$, where instead of $X$ we take $\Delta^{*}$.

Note that the complexes $\Delta^{n}$ are acyclic and hence the operad $E^{\Delta}$ is also acyclic.

Theorem 1. On the chain complex $C_{*}(X)$ of a topological space $X$ there exists a natural $E^{\Delta}$-coalgebra structure $\tau: C_{*}(X) \rightarrow \bar{E}^{\Delta}\left(C_{*}(X)\right)$ with the following universal property: if for some operad $\mathcal{E}$ there is a natural $\mathcal{E}$-coalgebra structure $\widetilde{\tau}: C_{*}(X) \rightarrow \overline{\mathcal{E}}\left(C_{*}(X)\right)$, then there exist a unique operad mapping $\xi: \mathcal{E} \rightarrow E^{\Delta}$ such that the following diagram commutes

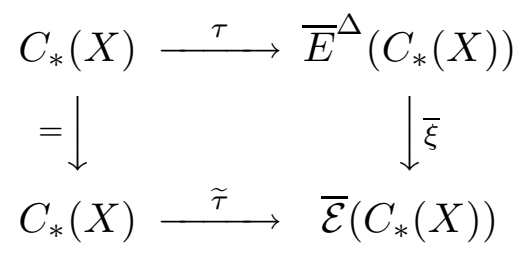

Proof. Our aim is to define natural operations

$$
\tau: E^{\Delta}(j) \otimes C_{*}(X) \rightarrow C_{*}(X)^{\otimes j} .
$$

Let $x_{n} \in C_{n}(X), y \in E^{\Delta}(j)=\operatorname{Hom}\left(\Delta^{*} ;\left(\Delta^{*}\right)^{\otimes j}\right)$. The element $x_{n} \in C_{n}(X)$ determines a chain mapping $\bar{x}_{n}: \Delta^{n} \rightarrow C_{*}(X)$ such that the generator $u_{n} \in \Delta^{n}$ maps to $x_{n}$.

Note that there is the operation $\tau^{n}: E^{\Delta}(j) \otimes \Delta^{n} \rightarrow\left(\Delta^{n}\right)^{\otimes j}$. Define the required operation $\tau$ by putting

$$
\tau\left(y \otimes x_{n}\right)=\left(\bar{x}_{n}\right)^{\otimes j} \circ \tau^{n}\left(y \otimes u_{n}\right) .
$$

Then we have the following commutative diagram

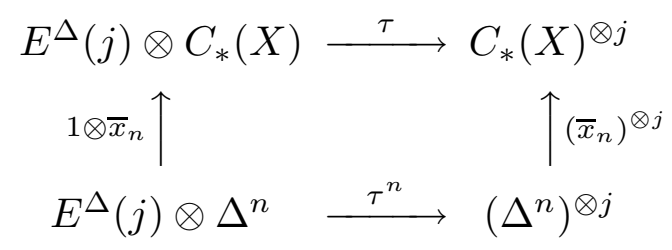


An $E^{\Delta}$-coalgebra structure on the chain complex $C_{*}(X)$ of a topological space $X$ induces an $E^{\Delta}$-algebra structure on the cochain complex

$$
C^{*}(X)=\operatorname{Hom}\left(C_{*}(X) ; R\right) .
$$

The corresponding operations $\mu: E^{\Delta}(j) \otimes C^{*}(X)^{\otimes j} \rightarrow C^{*}(X)$ are defined by the formulas

$$
\mu\left(y \otimes f_{1} \otimes \cdots \otimes f_{j}\right)(x)=\left(f_{1} \otimes \cdots \otimes f_{j}\right) \circ \tau(y \otimes x)
$$

where $y \in E^{\Delta}(j), f_{i}: C_{*}(X) \rightarrow R, x \in C_{*}(X)$. So we have

Theorem 1'. On the cochain complex $C^{*}(X)$ of a topological space $X$ there exists a natural $E^{\Delta}$-algebra structure $\mu: E^{\Delta}\left(C^{*}(X)\right) \rightarrow C^{*}(X)$ with the following universal property: if for some operad $\mathcal{E}$, there is a natural $\mathcal{E}$-algebra structure $\widetilde{\mu}: \mathcal{E}\left(C^{*}(X)\right) \rightarrow C^{*}(X)$, then there exists a unique operad mapping $\xi: \mathcal{E} \rightarrow E^{\Delta}$ such that the following diagram commutes

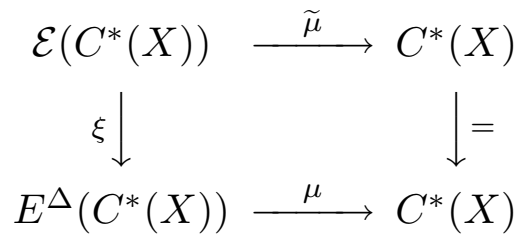

Let $R\left(\Sigma_{2}\right)$ be the $\Sigma_{2}$-free resolution with generators $e_{i}$ of dimensions $i$ and differential defined by the formula

$$
d\left(e_{i}\right)=e_{i-1}+(-1)^{i} e_{i-1} T, \quad T \in \Sigma_{2} .
$$

Since $E^{\Delta}(2)$ is acyclic, there is a $\Sigma_{2}$-chain mapping $R\left(\Sigma_{2}\right) \rightarrow E^{\Delta}(2)$ and hence a mapping

$$
R\left(\Sigma_{2}\right) \otimes \Sigma_{2} C^{*}(X)^{\otimes 2} \rightarrow C^{*}(X) .
$$

Its restriction on the elements $e_{i}$ is usually denoted by

$$
\cup_{i}: C^{*}(X) \otimes C^{*}(X) \rightarrow C^{*}(X)
$$

and called cup- $i$ product.

Let $p^{n}: \Delta^{n} \rightarrow S \Delta^{n-1}$ be the projection obtained by contracting the $(n-1)$ dimensional face spanned by the vertices with numbers $0,1, \ldots, n-1$. These projections induce the projection of operads $E^{\Delta} \rightarrow S E^{\Delta}$. For a topological space $X$, the suspension $S C_{*}(X)$ will be a coalgebra over the operad $S E^{\Delta}$. The following diagram commutes

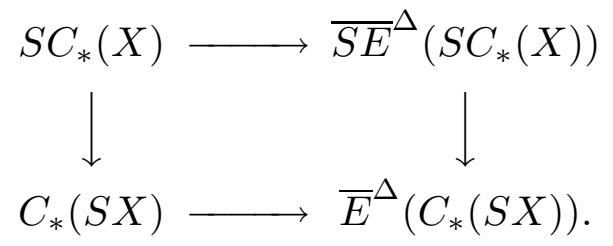

Iterating this construction we obtain the projections $E^{\Delta} \rightarrow S^{n} E^{\Delta}$ and the commutative diagrams

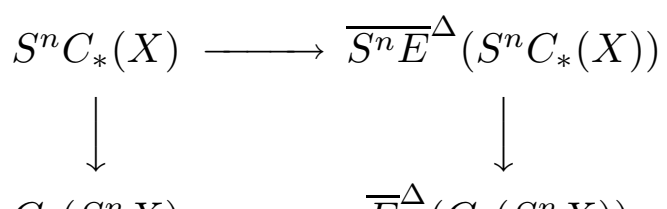


Note that the operad $E^{\Delta}$ may be not $\Sigma$-free and so it is not an $E_{\infty}$-operad. To obtain an $E_{\infty}$-operad we consider the operad $E^{\Delta} \otimes E \Sigma$ and denote it simply by $E$.

The projection $E \rightarrow E^{\Delta}$ induces an $E$-coalgebra structure on the chain complex $C_{*}(X)$ of a topological space $X$. The mapping $E^{\Delta} \rightarrow S E^{\Delta}$ induces the operad mapping $E \rightarrow S E$ and for the chain complex $C_{*}\left(S^{n} X\right)$ there is the corresponding commutative diagram similar to the diagram for the operad $E^{\Delta}$.

\section{$\S 3$. Bar and cobar constructions over operads}

Let $\mathcal{E}$ be an operad with right action $\nu: \mathcal{F} \times \mathcal{E} \rightarrow \mathcal{F}$ on a symmetric family $\mathcal{F}$, $\nu: \mathcal{F} \times \mathcal{E} \rightarrow \mathcal{F}$, and let $X$ be an algebra over the operad $\mathcal{E}$, given by $\mu: \mathcal{E}(X) \rightarrow X$.

Consider the simplicial object (monadic bar construction)

$$
B_{*}(\mathcal{F}, \mathcal{E}, X)=\left\{B_{n}(\mathcal{F}, \mathcal{E}, X)\right\}
$$

for which $B_{n}(\mathcal{F}, \mathcal{E}, X)=\mathcal{F E}^{n}(X)$ with face and degeneracy operators given by the formulas

$$
\begin{gathered}
d_{0}=\nu 1^{n}, d_{i}=1^{i} \gamma 1^{n-i}, 0<i<n ; \\
d_{n}=1^{n} \mu, s_{j}=1^{j+1} i 1^{n-j+1}, 0 \leq j \leq n .
\end{gathered}
$$

Its realization

$$
B(\mathcal{F}, \mathcal{E}, X)=\left|B_{*}(\mathcal{F}, \mathcal{E}, X)\right|=\sum_{n} \Delta^{n} \otimes B_{n}(\mathcal{F}, \mathcal{E}, X) / \sim
$$

is called the bar construction.

In the case of trivial $\mathcal{F}$, i.e. $\mathcal{F}(1)=R$ and $\mathcal{F}(j)=0$ if $j \geq 2$, the corresponding bar construction is denoted by $B(\mathcal{E}, X)$.

If $X$ is an $A$-algebra, i.e. simply an algebra, then the bar construction $B(A, X)$ will be chain equivalent to the desuspension of the usual bar construction $B(X)$, i.e.

$$
B(A, X) \simeq S^{-1} B X,[8] .
$$

Dually, if $X$ is a coalgebra $\tau: X \rightarrow^{M} \overline{\mathcal{E}}(X)$ over the operad $\mathcal{E}$, then we can consider the cosimplicial object $F^{*}(\mathcal{F}, \mathcal{E}, X)=\left\{F^{n}(\mathcal{F}, \mathcal{E}, X)\right\}$ for which $F^{n}(\mathcal{F}, \mathcal{E}, X)=$ $\overline{\mathcal{F E}}^{n}(X)$ with coface and codegeneracy operators given by the formulas

$$
\begin{gathered}
\delta^{0}=\bar{\nu} 1^{n}, \delta^{i}=1^{i} \bar{\gamma} 1^{n-i}, 0<i<n ; \\
\delta^{n}=1^{n} \tau, \sigma^{j}=1^{j+1} p 1^{n-j+1}, 0 \leq j \leq n .
\end{gathered}
$$

Its realization

$$
F(\mathcal{F}, \mathcal{E}, X)=\left|F^{*}(\mathcal{F}, \mathcal{E}, X)\right|=\operatorname{Hom}\left(\Delta^{*} ; F^{*}(\mathcal{F}, \mathcal{E}, X)\right)
$$

where Hom is considered in the category of cosimplicial objects, is called the cobar construction. 
If $X$ is an $A$-coalgebra, i.e. simply a coalgebra then the cobar construction $F(A, X)$ will be chain equivalent to the suspension of the usual Adams cobar construction $F(X)$, i.e.

$$
F(A, X) \simeq S F X,[8]
$$

Let now $X$ be an $n$-connected topological space. As was shown above, on the chain complex $S^{n} C_{*}\left(\Omega^{n} X\right)$ there is the $S^{n} E$-coalgebra structure

$$
\tau: S^{n} C_{*}\left(\Omega^{n} X\right) \rightarrow \overline{S^{n} E}\left(S^{n} C_{*}\left(\Omega^{n} X\right)\right)
$$

This structure and the mapping $j: S^{n} \Omega^{n} X \rightarrow X$ induce the mapping

$$
S^{n} C_{*}\left(\Omega^{n} X\right) \rightarrow \overline{S^{n} E}\left(S^{n} C_{*}\left(\Omega^{n} X\right)\right) \rightarrow \overline{S^{n} E}\left(C_{*}(X)\right)
$$

This mapping is a coaugmentation of the cosimplicial object $F^{*}\left(S^{n} E, E, C_{*}(X)\right)$ and hence it induces a mapping of $S^{n} E$-coalgebras:

$$
S^{n} C_{*}\left(\Omega^{n} X\right) \rightarrow F\left(S^{n} E, E, C_{*}(X)\right) .
$$

Theorem 2. For any $n$-connected topological space $X$, the mapping

$$
S^{n} C_{*}\left(\Omega^{n} X\right) \rightarrow F\left(S^{n} E, E, C_{*}(X)\right)
$$

is a chain equivalence of $S^{n} E$-coalgebras.

Proof. For $n=1$ the chain equivalence $S C_{*}(\Omega X) \rightarrow F\left(S E, E, C_{*}(X)\right)$ follows from the Adams chain equivalence

$$
S C_{*}(\Omega X) \simeq S F C_{*}(X)
$$

and from the chain equivalence

$$
S F C_{*}(X) \simeq F\left(S E, E, C_{*}(X)\right),[8]
$$

Suppose that for any $(n-1)$-connected topological space $X$, we have a chain equivalence

$$
S^{n-1} C_{*}\left(\Omega^{n-1} X\right) \rightarrow F\left(S^{n-1} E, E, C_{*}(X)\right) .
$$

Then for an $n$-connected topological space $X$ we will have the following sequence of chain equivalences

$$
\begin{gathered}
S^{n} C_{*}\left(\Omega^{n} X\right) \simeq S F\left(S^{n-1} E, E, C_{*}(\Omega X)\right) \simeq F\left(S^{n} E, S E, S C_{*}(\Omega X)\right) \simeq \\
F\left(S^{n} E, S E, F\left(S E, E, C_{*}(X)\right)\right) \simeq F\left(S^{n} E, E, C_{*}(X)\right) .
\end{gathered}
$$

The composition will be the desirable chain equivalence

$$
S^{n} C_{*}\left(\Omega^{n} X\right) \simeq F\left(S^{n} E, E, C_{*}(X)\right)
$$


Let $X$ be an $E$-coalgebra. Consider the spectral sequence of the cobar construction $F\left(S^{n} E, E, X\right)$ with respect to the filtration determined by the operad grading. Namely, for any operad $\mathcal{E}$ we define the grading of mappings $f: \mathcal{E}(j) \rightarrow X^{\otimes j}$ to be equal to $j$.

Thus we will have the grading of the elements of $\overline{S^{n} E}(X), \overline{S^{n} E} \circ \bar{E}(X)$ and so on. Similarly we will have the grading of the elements of the cobar construction $F\left(S^{n} E, E, X\right)$ which determines in it a decreasing filtration.

Note that the mappings $\bar{\mu}: \overline{S^{n} E} \rightarrow \overline{S^{n} E} \circ \bar{E}, \bar{\gamma}: \bar{E} \rightarrow \bar{E} \circ \bar{E}$ preserve gradings and the mapping $\tau: X \rightarrow \bar{E}(X)$ increases it. Therefore the first term of the corresponding spectral sequence will be isomorphic to the homology of the cobar construction $F\left(S^{n} E, E, X\right)$, where $X$ is considered as a trivial $E$-coalgebra.

To calculate its homology, recall the notion of the iterated cobar construction over a cocommutative coalgebra $K$.

The cobar construction $F K$ will be a cocommutative Hopf algebra with a coproduct $\nabla: F K \rightarrow F K \otimes F K$ defined on the generators $[x] \in F K$ by the formula

$$
\nabla[x]=[x] \otimes 1+1 \otimes[x]
$$

Note that a commutative coalgebra $K$ may be considered as an $E$-coalgebra. The required $E$-coalgebra structure is induced by the projection $E \rightarrow E_{0}$. Moreover, the chain equivalence $S F K \simeq F(S E, E, K)$ will be a chain equivalence of $S E$ coalgebras.

Thus, in this case the Adams cobar construction may be iterated and there are chain equivalences

$$
S^{n} F^{n} K \simeq F\left(S^{n} E, E, K\right)
$$

In particular, if a chain complex $X$ has a trivial $E$-coalgebra structure then there are the chain equivalences

$$
S^{n} F^{n} X \simeq F\left(S^{n} E, E, X\right)
$$

So to calculate the homology of the cobar construction $F\left(S^{n} E, E, X\right)$ of a trivial $E$-coalgebra $X$, it is sufficient to calculate the homology of the iterated Adams cobar construction $F^{n} X$.

Consider the case of $Z / 2$-coefficients. The homology $H_{*}(F X)$ is isomorphic to the tensor algebra $T S^{-1} H_{*}(X)$ on the desuspension $S^{-1} H_{*}(X)$ of $H_{*}(X)$, i.e.

$$
H_{*}(F X) \cong T S^{-1} H_{*}(X) .
$$

The double cobar construction $F^{2} X$ will be chain equivalent to the cobar construction $F T S^{-1} H_{*}(X)$, where $T S^{-1} H_{*}(X)$ is considered as a Hopf algebra with a coproduct

$$
\nabla: T S^{-1} H_{*}(X) \rightarrow T S^{-1} H_{*}(X) \otimes T S^{-1} H_{*}(X)
$$

determined on the generators $[x] \in S^{-1} H_{*}(X)$ by the formula

$$
\nabla[x]=[x] \otimes 1+1 \otimes[x] .
$$

For a graded module $M$ (over $Z / 2$ ) denote by $L M$ the free Lie algebra generated by $M$ and by $T_{s} M$ the quotient of the tensor algebra $T M$ over the permutations 
The Hopf algebra structure on $T M$ induces the Hopf algebra structure on $T_{s} M$. By the Poincare-Birkhoff-Witt theorem there is an isomorphism of coalgebras

$$
T M \cong T_{s} L M
$$

and hence there is an isomorphism

$$
T S^{-1} H_{*}(X) \cong T_{s} L S^{-1} H_{*}(X) \text {. }
$$

For a coalgebra $K$ denote by $P K$ the module of it's primitive elements, i.e.

$$
P K=\{x \in K \mid \nabla(x)=x \otimes 1+1 \otimes x\} .
$$

Note that the module $P T_{s} M$ of primitive elements of the Hopf algebra $T_{s} M$ is generated by the elements of the form $x^{2^{k}}, x \in M, k \geq 0$.

The homology of $F T_{s} M$ is isomorphic to the free commutative algebra generated by the module $S^{-1} P T_{s} M$ and hence there is an isomorphism

$$
H_{*}\left(F^{2} X\right) \cong T_{s} S^{-1} P T_{s} L S^{-1} H_{*}(X) .
$$

So this construction may be iterated and by induction we obtain isomorphisms

$$
H_{*}\left(F^{n} X\right) \cong T_{s}\left(S^{-1} P T_{s}\right)^{n-1} L S^{-1} H_{*}(X)
$$

and hence isomorphisms

$$
S^{-n} H_{*}\left(F\left(S^{n} E, E, X\right)\right) \cong T_{s}\left(S^{-1} P T_{s}\right)^{n-1} L S^{-1} H_{*}(X) .
$$

Recall that a graded module $L$ (over $Z / 2$ ) is called an $n$-Lie algebra if there is given an operation [, ]: $L \otimes L \rightarrow L$ called an $n$-Lie bracket of dimension $n$ and satisfying the relations

$$
\begin{gathered}
{[x, x]=0} \\
{[x, y]+[y, x]=0} \\
{[x,[y, z]]=[[x, y], z]+[y,[x, z]] .}
\end{gathered}
$$

For a graded module $M$ denote by $\mathcal{E}_{n} M$ the module generated by the elements $e_{i_{1}} \ldots e_{i_{k}} x$, where $x \in M, 0 \leq i_{1} \leq \cdots \leq i_{k} \leq n$, and the dimensions of these elements are defined equal to $i_{1}+2 i_{2}+\cdots+2^{k-1} i_{k}+2^{k} \operatorname{dim}(x)$.

These elements $e_{i_{1}} \ldots e_{i_{k}} x$ of $\mathcal{E}_{n} M$ may be rewritten using the Dyer-Lashof algebra $\mathcal{R}$ in the form

$$
Q^{j_{1}} \ldots Q^{j_{k}} x ; \quad j_{1} \leq 2 j_{2}, \ldots, j_{k-1} \leq 2 j_{k}, \operatorname{dim}(x) \leq j_{k} \leq \operatorname{dim}(x)+n,
$$

where

$$
\begin{gathered}
j_{k}=i_{k}+\operatorname{dim}(x), \\
j_{k-1}=i_{k-1}+i_{k}+2 \operatorname{dim}(x),
\end{gathered}
$$


It is clear that the sequences $Q^{j_{1}} \ldots Q^{j_{k}}$ are admissible and represent elements of the Dyer-Lashof algebra $\mathcal{R},[5],[14]$.

Denote by $\mathcal{R}_{n} M$ the submodule of $\mathcal{R} \otimes M$ generated by the elements

$$
Q^{j_{1}} \ldots Q^{j_{k}} \otimes x
$$

where $Q^{j_{1}} \ldots Q^{j_{k}}$, the admissible sequences with $\operatorname{dim}(x) \leq j_{k} \leq \operatorname{dim}(x)+n$. Then there is an isomorphism $\mathcal{E}_{n} M \cong \mathcal{R}_{n} M$.

The correspondence $M \longmapsto \mathcal{R}_{n} M$ determines the monad $\mathcal{R}_{n}$ in the category of graded modules. Algebras over the monad $\mathcal{R}_{n}$ we will call $\mathcal{R}_{n}$-modules.

For an $n$-Lie algebra $L_{n}$ we will have the module $\mathcal{R}_{n} L_{n}$. Denote also by $T_{s} \mathcal{R}_{n} L_{n}$ the quotient algebra of the free commutative algebra generated by the module $\mathcal{R}_{n} L_{n}$ modulo the relations $x \cdot x=e_{0} x$.

From the above considerations it follows that if $X$ is a chain complex (over $Z / 2$ ) considered as the trivial $E$-coalgebra, then there are isomorphisms

$$
S^{-n} H_{*}\left(F\left(S^{n} E, E, X\right)\right) \cong T_{s} \mathcal{R}_{n-1} L_{n-1} S^{-n} H_{*}(X) \text {. }
$$

Hence we have

Theorem 3. If $X$ is an n-connected topological space then there is the spectral sequence which converges to $H_{*}\left(\Omega^{n} X\right)$ and for the first term of this spectral sequence (over $Z / 2$ ) there is the isomorphism

$$
E^{1} \cong T_{s} \mathcal{R}_{n-1} L_{n-1} S^{-n} H_{*}(X)
$$

Define the notion of a $\mathcal{P}_{n}$-algebra with $Z / 2$-coefficients generalizing the notion of an $n$-Poisson algebra.

A graded module $M$ (over $Z / 2$ ) will be called a $\mathcal{P}_{n}$-algebra if

1. There is given a structure of a commutative algebra

$$
x \otimes y \longmapsto x \cdot y, \quad x, y \in M .
$$

2. There is given a structure of an $n$-Lie algebra

$$
x \otimes y \longmapsto[x, y], \quad x, y \in M,
$$

and the $n$-Lie algebra structure with the commutative algebra structure form an $n$-Poisson algebra structure.

3. There is given a structure of $\mathcal{R}_{n}$-module

$$
\mathcal{R}_{n} M \rightarrow M
$$

compatible with the $n$-Poisson algebra structure [4].

Denote by $\mathcal{P}_{n}$ the monad which associates to a graded module $M$ the free $\mathcal{P}_{n^{-}}$ algebra generated by $M$. Then there is an isomorphism

$$
\mathcal{P}_{n}(M) \cong T_{s} \mathcal{R}_{n} L_{n} M
$$


Theorem 4. The first term of the considered spectral sequence of $H_{*}\left(\Omega^{n} X\right)$ (over $Z / 2$ ) is isomorphic to the free $\mathcal{P}_{n-1}$-algebra generated by $S^{-n} H_{*}(X)$, i.e.

$$
E^{1} \cong \mathcal{P}_{n-1} S^{-n} H_{*}(X)
$$

Consider now $Z / p$-coefficients, $p>2$. Note that the module $P T_{s} M$ of primitive elements of the Hopf algebra $T_{s} M$ in this case is generated by the elements $x \in M$ and the elements $x^{p^{k}}$ for which $k>0$ and $\operatorname{dim}(x)$ is even.

However, the homology $H_{*}\left(F T_{s} M\right)$ is generated not only by the primitive elements of $T_{s} M$ but also by the elements $\beta x^{p^{k}}$ where $\beta$ is the Bockstein homomorphism. Denote the module generated by the primitive elements and the elements $\beta x^{p^{k}}$ by $P_{\beta} T_{s} M$.

Then the homology of $F T_{s} M$ will be isomorphic to $T_{s} S^{-1} P_{\beta} T_{s} M$.

Thus for the homology $H_{*}\left(F^{n} X\right)$ of the iterated cobar constructions $F^{n} X$ over $Z / p$-coefficients there are the isomorphisms

$$
H_{*}\left(F^{n} X\right) \cong T_{s}\left(S^{-1} P_{\beta} T_{s}\right)^{n-1} L S^{-1} H_{*}(X)
$$

and hence the isomorphisms

$$
S^{-n} H_{*}\left(F\left(S^{n} E, E, X\right)\right) \cong T_{s}\left(S^{-1} P_{\beta} T_{s}\right)^{n-1} L S^{-1} H_{*}(X) .
$$

Recall that a graded module $L$ (over $Z / p$ ) is called an $n$-Lie algebra if there is given an operation [, ]: $L \otimes L \rightarrow L$ of dimension $n$, called an $n$-Lie bracket and satisfying the relations

$$
\begin{gathered}
{[x, y]+(-1)^{\epsilon}[y, x]=0} \\
{[x,[y, z]]=[[x, y], z]+(-1)^{\epsilon}[y,[x, z]]}
\end{gathered}
$$

where $\epsilon=(\operatorname{dim}(x)+n) \cdot(\operatorname{dim}(y)+n)$.

For a graded module $M$ denote by $\mathcal{E}_{n}(M)$ the module generated by the sequences $\beta^{\epsilon_{1}} e_{i_{1}} \ldots \beta^{\epsilon_{k}} e_{i_{k}} x$, where $x \in M, \epsilon=0,1,0 \leq i_{1} \leq \cdots \leq i_{k} \leq n$.

For the elements $e_{i} y$ it is demanded that $i$ and $\operatorname{dim}(y)$ have the same parity and the dimensions of the elements $e_{i} y$ are defined equal to $p \cdot \operatorname{dim}(y)+(p-1) i$.

These elements $\beta^{\epsilon_{1}} e_{i_{1}} \ldots \beta^{\epsilon_{k}} e_{i_{k}} x$, may be rewritten using the mod-p Dyer-Lashof algebra $\mathcal{R}$ in the form $\beta^{\epsilon_{1}} Q^{j_{1}} \ldots \beta^{\epsilon_{k}} Q^{j_{k}} x$, where

$$
\begin{gathered}
2 j_{k}=i_{k}+\operatorname{dim}(x), \\
2 j_{k-1}=i_{k-1}+(p-1) i_{k}+p \operatorname{dim}(x)-\epsilon_{k}, \\
\cdots \\
2 j_{1}=i_{1}+(p-1) i_{2}-\epsilon_{2}+\cdots+p^{k-1} \operatorname{dim}(x) .
\end{gathered}
$$

It is clear that the sequences $\beta^{\epsilon_{1}} Q^{j_{1}} \ldots \beta^{\epsilon_{k}} Q^{j_{k}}$ are admissible and represent elements of the Dyer-Lashof algebra $\mathcal{R}$.

Denote by $\mathcal{R}_{n} M$ the submodule of $\mathcal{R} \otimes M$ generated by the elements 
where $\beta^{\epsilon_{1}} Q^{j_{1}} \ldots \beta^{\epsilon_{k}} Q^{j_{k}}$ are admissible sequences with $\operatorname{dim}(x) \leq 2 j_{k} \leq \operatorname{dim}(x)+n$. Then there is an isomorphism $\mathcal{E}_{n} M \cong \mathcal{R}_{n} M$.

The correspondence $M \longmapsto \mathcal{R}_{n} M$ determines the monad $\mathcal{R}_{n}$ in the category of graded $Z / p$-modules. Algebras over the monad $\mathcal{R}_{n}$ we will call $\mathcal{R}_{n}$-modules.

For an $n$-Lie algebra $L_{n}$ we have the module $\mathcal{R}_{n} L_{n}$. Denote also by $T_{s} \mathcal{R}_{n} L_{n}$ the quotient algebra of the free commutative algebra generated by the module $\mathcal{R}_{n} L_{n}$ modulo the relations $e_{0} x=x^{p}$.

From the above considerations follows that if $X$ is a chain complex (over $Z / p$ ) considered as a trivial $E$-coalgebra, then there are the isomorphisms

$$
S^{-n} H_{*}\left(F\left(S^{n} E, E, X\right)\right) \cong T_{s} \mathcal{R}_{n-1} L_{n-1} S^{-n} X_{*}
$$

Hence we have

Theorem 3'. For the first term of the considered spectral sequence of $H_{*}\left(\Omega^{n} X\right)$ (over $Z / p$ ), there is an isomorphism

$$
E^{1} \cong T_{s} \mathcal{R}_{n-1} L_{n-1} S^{-n} H_{*}(X)
$$

A graded module $M$ (over $Z / p$ ) will be called a $\mathcal{P}_{n}$-algebra if

1. There is given a structure of a commutative algebra

$$
x \otimes y \longmapsto x \cdot y, \quad x, y \in M
$$

2. There is given a structure of an $n$-Lie algebra

$$
x \otimes y \longmapsto[x, y], \quad x, y \in M
$$

and the $n$-Lie algebra structure with the commutative algebra structure form an $n$-Poisson algebra structure.

3. There is given a structure of an $\mathcal{R}_{n}$-module

$$
\mathcal{R}_{n} M \rightarrow M
$$

compatible with the $n$-Poisson algebra structure [4].

Denote by $\mathcal{P}_{n}$ the monad which associates to a graded module $M$ the free $\mathcal{P}_{n^{-}}$ algebra generated by $M$. Then there is an isomorphism

$$
\mathcal{P}_{n}(M) \cong T_{s} \mathcal{R}_{n} L_{n} M
$$

and hence the Theorem 3' may be reformulated

Theorem 4'. The first term of the considered spectral sequence of $H_{*}\left(\Omega^{n} X\right)$ (over $Z / p$ ) is isomorphic to the free $\mathcal{P}_{n-1}$-algebra generated by $S^{-n} H_{*}(X)$, i.e.

$$
E^{1} \cong \mathcal{P}_{n-1} S^{-n} H_{*}(X)
$$

Note that in the case of characteristic zero coefficients the module $P T_{s} M$ of 
Theorem 3". For the first term of the considered spectral sequence of $H_{*}\left(\Omega^{n} X\right)$ (over a field of characteristic zero) there is an isomorphism

$$
E^{1} \cong T_{s} L_{n-1} S^{-n} H_{*}(X) .
$$

$\S 5$. The second term of the spectral sequence of the homology of iterated loop spaces

To determine the second term of the spectral sequence of the homology of iterated loop spaces, we consider the relation between the Steenrod algebra and the DyerLashof algebra.

We begin with $Z / 2$-coefficients. Let $\mathcal{A}$ be the mod-2 Steenrod algebra with $S q^{0} \neq 1$ and $\mathcal{K}$ the Milnor coalgebra (dual to the Steenrod algebra). We will consider $\mathcal{K}$ as a family $\mathcal{K}(m)$ of polynomial algebras $\mathcal{K}(m), m \geq 1$ generated by the elements $\xi_{i}(m), 1 \leq i \leq m$ of dimensions $2^{i}-1$.

Define products $\mathcal{K} \otimes \mathcal{K}, \mathcal{K} \times \mathcal{K}$ to be the families

$$
\begin{gathered}
(\mathcal{K} \otimes \mathcal{K})(m)=\mathcal{K}(m) \otimes \mathcal{K}(m), \\
(\mathcal{K} \times \mathcal{K})(m)=\sum_{k} \mathcal{K}(k) \otimes \mathcal{K}(m-k) .
\end{gathered}
$$

We denote the multiplication by $\pi: \mathcal{K} \otimes \mathcal{K} \rightarrow \mathcal{K}$.

The comultiplication $\nabla: \mathcal{K} \rightarrow \mathcal{K} \times \mathcal{K}$ is defined on the generators by the formula

$$
\nabla\left(\xi_{i}(m)\right)=\sum_{j, k} \xi_{i-j}^{2^{j}}(k) \otimes \xi_{j}(m-k) .
$$

On the other elements the comultiplication is determined by the Hopf relation. So we can consider $\mathcal{K}$ as a Hopf algebra.

Let now $\mathcal{R}$ be the Dyer-Lashof algebra. Define a homomorphism $\varphi: \mathcal{K} \rightarrow \mathcal{R}$ of dimension -1 by putting

$$
\varphi(x)= \begin{cases}Q^{i-1}, & x=\xi_{1}^{i}(1), \\ 0, & \text { otherwise } .\end{cases}
$$

It is easy to see that $\varphi \cup \varphi=0$ and hence $\varphi$ is a twisting cochain.

Denote by $\bar{E}_{*}$ the comonad in the category of graded modules which associates to a graded module $M$ the graded module $H_{*}(\bar{E}(M))$. The $E$-coalgebra structure on the chain complex $C_{*}(X)$ of a topological space $X$ induces on its homology $H_{*}(X)$ the $\bar{E}_{* \text {-coalgebra structure. }}$

This structure consists of the commutative coalgebra structure

$$
\nabla_{*}: H_{*}(X) \rightarrow H_{*}(X) \otimes H_{*}(X)
$$

and of the coaction

$$
\tau: H_{*}(X) \rightarrow \mathcal{K} \otimes H_{*}(X),
$$


For an $n$-connected space $X$, these structures induce on $\mathcal{P}_{n-1} S^{-n} H_{*}(X)$ a differential $d_{\varphi}$ defined on the generators $s^{-n} x_{i+n} \in H_{i+n}(X)$ by the formula

$$
d_{\varphi}\left(s^{-n} x_{i+n}\right)=\sum_{x^{\prime}<x^{\prime \prime}}\left[s^{-n} x^{\prime}, s^{-n} x^{\prime \prime}\right]+\varphi \cap s^{-n} x_{i+n}
$$

Denote the corresponding differential $\mathcal{P}_{n-1}$-algebra by $\mathcal{P}_{n-1 \varphi} S^{-n} H_{*}(X)$. Then we will have

Theorem 5. For the second term of the spectral sequence of $H_{*}\left(\Omega^{n} X\right)$ (over $Z / 2$ ) there is an isomorphism

$$
E^{2} \cong H_{*}\left(\mathcal{P}_{n-1 \varphi} S^{-n} H_{*}(X)\right)
$$

Consider now the relation between the mod-p Steenrod algebra and the mod-p Dyer-Lashof algebra. Let $\mathcal{A}$ be the mod-p Steenrod algebra with $S q^{0} \neq 1$ and $\mathcal{K}$ the Milnor coalgebra (dual to the Steenrod algebra). We will consider $\mathcal{K}$ as a family $\mathcal{K}(m)$ of polynomial algebras $\mathcal{K}(m), m \geq 1$ generated by the elements $\xi_{i}(m), \tau_{i}(m)$, $1 \leq i \leq m$ of dimensions $2\left(p^{i}-1\right)$ and $2 p^{i}-1$ correspondingly.

There are a multiplication

$$
\pi: \mathcal{K} \otimes \mathcal{K} \rightarrow \mathcal{K}
$$

and a comultiplication

$$
\nabla: \mathcal{K} \rightarrow \mathcal{K} \times \mathcal{K}
$$

defined on the generators by the formulas

$$
\begin{gathered}
\nabla\left(\xi_{i}(m)\right)=\sum_{j, k} \xi_{i-j}^{p^{j}}(k) \otimes \xi_{j}(m-k) . \\
\nabla\left(\tau_{i}(m)\right)=\tau_{i}(m) \otimes 1+\sum_{j, k} \xi_{i-j}^{p^{j}}(k) \otimes \tau_{j}(m-k) .
\end{gathered}
$$

On the other elements the comultiplication is determined by the Hopf relation. So we can consider $\mathcal{K}$ as a Hopf algebra.

Let now $\mathcal{R}$ be the mod-p Dyer-Lashof algebra. Define the homomorphism $\varphi: \mathcal{K} \rightarrow$ $\mathcal{R}$ of dimension -1 by putting

$$
\varphi(x)= \begin{cases}\beta P^{i}, & x=\xi_{1}^{i}(1), \\ P^{i}, & x=\xi_{1}^{i-1}(1) \tau_{1}(1), \\ 0, & \text { otherwise. }\end{cases}
$$

It is easy to see that $\varphi \cup \varphi=0$ and hence $\varphi$ is the twisting cochain.

Denote by $\bar{E}_{*}$ the comonad in the category of graded $Z / p$-modules which associates to a graded module $M$ the graded module $H_{*}(\bar{E}(M))$. The $E$-coalgebra structure on the chain complex $C_{*}(X)$ of a topological space $X$ induces on its homology $H_{*}(X)$ an $\bar{E}_{*}$-coalgebra structure.

This structure consists of the commutative coalgebra structure 
and the coaction of the Milnor coalgebra

$$
H_{*}(X) \rightarrow \mathcal{K} \otimes H_{*}(X)
$$

For an $n$-connected space $X$, these structures induce on $\mathcal{P}_{n-1} S^{-n} H_{*}(X)$ a differential $d_{\varphi}$ defined on the generators $s^{-n} x_{i+n} \in H_{i+n}(X)$ by the formula

$$
d_{\varphi}\left(s^{-n} x_{i+n}\right)=\sum_{x^{\prime}<x^{\prime \prime}}(-1)^{\epsilon}\left[s^{-n} x^{\prime}, s^{-n} x^{\prime \prime}\right]+\varphi \cap s^{-n} x_{i+n}
$$

where $\sum x^{\prime} \otimes x^{\prime \prime}=\nabla_{*}\left(x_{i+n}\right), \epsilon=n \cdot \operatorname{dim}\left(x^{\prime}\right)$.

Denote the corresponding differential $\mathcal{P}_{n-1}$-algebra by $\mathcal{P}_{n-1 \varphi} S^{-n} H_{*}(X)$. Then we have

Theorem 5'. For the second term of the considered spectral sequence of $H_{*}\left(\Omega^{n} X\right)$ (over $\left.Z / p\right)$ there is an isomorphism

$$
E^{2} \cong H_{*}\left(\mathcal{P}_{n-1 \varphi} S^{l-n} H_{*}(X)\right)
$$

Note that in the case of characteristic zero coefficients, the $\bar{E}_{*}$-coalgebra structure on the homology $H_{*}(X)$ of a topological space $X$ consists only of the commutative coalgebra structure

$$
\nabla_{*}: H_{*}(X) \rightarrow H_{*}(X) \otimes H_{*}(X)
$$

Hence we have

Theorem 5". For the second term of the considered spectral sequence of $H_{*}\left(\Omega^{n} X\right)$ (over a field of characteristic zero), there is an isomorphism

$$
E^{2} \cong H_{*}\left(P_{n-1 \varphi} S^{-n} H_{*}(X)\right)
$$

where the differential $d_{\varphi}$ is defined on the generators $s^{-n} x_{i+n} \in H_{i+n}(X)$ by the formula

$$
d_{\varphi}\left(s^{-n} x_{i+n}\right)=\sum_{x^{\prime}<x^{\prime \prime}}(-1)^{\epsilon}\left[s^{-n} x^{\prime}, s^{-n} x^{\prime \prime}\right],
$$

where $\sum x^{\prime} \otimes x^{\prime \prime}=\nabla_{*}\left(x_{i+n}\right), \epsilon=n \cdot \operatorname{dim}\left(x^{\prime}\right)$.

$\S 6$. The homology of iterated loop spaces of the real projective spaces

Some of the most important spaces in Algebraic Topology, besides spheres, are the real projective spaces $R P^{n}$ and $R P^{\infty}$. In some sense these spaces are opposite to spheres. The homology $H_{*}\left(R P^{\infty}\right)$ (over $\left.Z / 2\right)$ is the free $\bar{E}_{*}$-coalgebra with one 1dimensional generator, whereas for spheres that homology is a trivial $\bar{E}_{*}$-coalgebra.

On the other hand, the Adams spectral sequences of stable homotopy groups of $R P^{\infty}$ and $R P^{\infty} / R P^{n}$ is very similar to the corresponding spectral sequence of spheres; it is a problem to find the relations between them.

The homology of iterated loop spaces gives an approximation of the homotopy groups. Here we consider the problem of calculation of the homology 
of the iterated loop spaces of the space $R P^{\infty} / R P^{n}$ with $Z / 2$ coefficients.

Denote the $i$-dimensional generator of $H_{*}\left(R P^{\infty}\right)$ as $e_{i}$. A coalgebra structure on these generators is determined by the formula

$$
\nabla\left(e_{i}\right)=\sum_{j} e_{j} \otimes e_{i-j} .
$$

An action of the Steenrod algebra is determined by the formula

$$
S q^{j}\left(e_{i}\right)=\left(\begin{array}{c}
i-j \\
j
\end{array}\right) e_{i-j}
$$

Usually, on the homology $H_{*}(X)$ of a topological space $X$ (over $Z / 2$ ) there is not only $\bar{E}_{*}$-coalgebra structure. Besides that there are functional homology operations

$$
\tau_{*}^{n}: H_{*}(X) \rightarrow \bar{E}_{*}^{n}\left(H_{*}(X)\right), \quad[15],[16] .
$$

These operations determine the higher differentials of the spectral sequence.

Fortunately, $H_{*}\left(R P^{\infty}\right)$ is a free $\bar{E}_{*}$-coalgebra and hence the higher functional homology operations are trivial. The homology $H_{*}\left(R P^{n}\right)$ is the $\bar{E}_{*}$-subcoalgebra of $H_{*}\left(R P^{\infty}\right)$ and hence the $\bar{E}_{*}$-coalgebra structure on $H_{*}\left(R P^{\infty}\right)$ induces an $\bar{E}_{*^{-}}$ coalgebra structure on

$$
H_{*}\left(R P^{\infty}\right) / H_{*}\left(R P^{n}\right) \cong H_{*}\left(R P^{\infty} / R P^{n}\right) .
$$

Therefore on the homology $H_{*}\left(R P^{\infty} / R P^{n}\right)$ there are no higher functional homology operations. So the spectral sequence of the homology of the iterated loop spaces of $R P^{\infty} / R P^{n}$ has no higher differentials and we have

Theorem 6. The homology $H_{*}\left(\Omega^{m}\left(R P^{\infty} / R P^{n}\right)\right), n \geq m$ (over $Z / 2$ ) is isomorphic to the homology of the differential $\mathcal{P}_{m-1}$-algebra $\mathcal{P}_{m-1 \varphi} S^{-m} H_{*}\left(R P^{\infty} / R P^{n}\right)$, where the differential $d_{\varphi}$ on the generators $s^{-m} e_{i}$ is defined by the formula

$$
d_{\varphi}\left(s^{-m} e_{i}\right)=\sum_{\substack{j \\
2 j<i}}\left[s^{-m} e_{j}, s^{-m} e_{i-j}\right]+\sum_{\substack{j \\
2 j \leq i}}\left(\begin{array}{c}
i-j \\
j
\end{array}\right) e_{2 j-i+m-1}\left(s^{-m} e_{i-j}\right) .
$$

Note that in the case $m=1$ we have the isomorphisms

$$
\mathcal{P}_{0} S^{-1} H_{*}\left(R P^{\infty} / R P^{n}\right) \cong T_{s} L S^{-1} H_{*}\left(R P^{\infty} / R P^{n}\right) \cong T S^{-1} H_{*}\left(R P^{\infty} / R P^{n}\right),
$$

and the differential in the tensor algebra $T S^{-1} H_{*}\left(R P^{\infty} / R P^{n}\right)$ on the generators $u_{i}=s^{-1} e_{i+1}$ has a very simple form:

$$
d_{\varphi}\left(u_{i}\right)=\sum_{j} u_{j} \otimes u_{i-j-1} .
$$

From here it follows that the homology $H_{*}\left(\Omega\left(R P^{\infty} / R P^{n}\right)\right)($ over $Z / 2)$ is isomorphic to the algebra generated by the elements $u_{i}, n \leq i \leq 2 n$, of dimensions $i$ and relations

$$
\begin{gathered}
u_{n} \cdot u_{n}=0 ; \\
u_{n} \cdot u_{n+1}+u_{n+1} \cdot u_{n}=0
\end{gathered}
$$


So, as a graded module, the homology $H_{*}\left(\Omega\left(R P^{\infty} / R P^{n}\right)\right)$ is generated by the noncommutative products $u_{n_{1}} \cdot \ldots \cdot u_{n_{k}}$ with $n \leq n_{1} \leq 2 n, n<n_{2}, \ldots, n_{k} \leq 2 n$.

Consider the homology $H_{*}\left(\Omega^{2}\left(R P^{\infty} / R P^{2}\right)\right)$. It is isomorphic to the homology of the differential $\mathcal{P}_{1}$-algebra $\mathcal{P}_{1 \varphi}\left\{u_{i} \mid i \geq 1\right\}$, where $u_{i}=s^{-2} e_{i+2}$ and the differential is determined by the formulas

$$
\begin{aligned}
& d_{\varphi}\left(u_{2 i+1}\right)=\sum_{j<i}\left[u_{j}, u_{2 i-j-1}\right]+\left(\begin{array}{c}
i+2 \\
i+1
\end{array}\right) e_{0}\left(u_{i}\right) \\
& d_{\varphi}\left(u_{2 i+2}\right)=\sum_{j<i}\left[u_{j}, u_{2 i-j}\right]+e_{1}\left(u_{i}\right) .
\end{aligned}
$$

In small dimensions we have

$$
\begin{aligned}
& d\left(u_{1}\right)=0 \\
& d\left(u_{2}\right)=0 \\
& d\left(u_{3}\right)=u_{1} u_{1} \\
& d\left(u_{4}\right)=e_{1}\left(u_{1}\right) \\
& d\left(u_{5}\right)=\left[u_{1}, u_{2}\right] \\
& d\left(u_{6}\right)=\left[u_{1}, u_{3}\right]+e_{1}\left(u_{2}\right) \\
& d\left(u_{7}\right)=\left[u_{1}, u_{4}\right]+\left[u_{2}, u_{3}\right]+u_{3} u_{3} ; \\
& d\left(u_{8}\right)=\left[u_{1}, u_{5}\right]+\left[u_{2}, u_{4}\right]+e_{1}\left(u_{3}\right) .
\end{aligned}
$$

From these formulas it follows that the homology $H_{i}=H_{i}\left(\Omega^{2}\left(R P^{\infty} / R P^{2}\right)\right)$ in small dimensions $i$ has the following generators

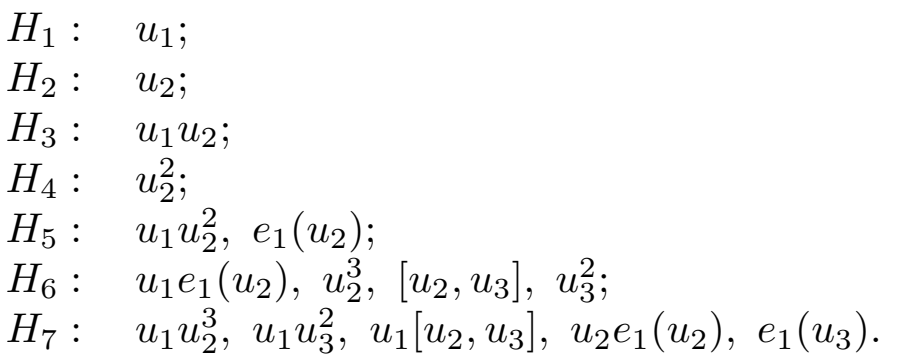

Consider the homology $H_{*}\left(\Omega^{3}\left(R P^{\infty} / R P^{3}\right)\right)$. It is isomorphic to the homology of the differential $\mathcal{P}_{2}$-algebra $\mathcal{P}_{2 \varphi}\left\{u_{i} \mid i \geq 1\right\}$, where $u_{i}=s^{-3} e_{i+3}$ and the differential is determined by the formulas

$$
\begin{aligned}
& d_{\varphi}\left(u_{2 i+1}\right)=\sum_{j<i-1}\left[u_{j}, u_{2 i-j-2}\right]+\left(\begin{array}{l}
i+3 \\
i+1
\end{array}\right) e_{0}\left(u_{i}\right)+e_{2}\left(u_{i-1}\right) \\
& d_{\varphi}\left(u_{2 i+2}\right)=\sum_{j \leq i-1}\left[u_{j}, u_{2 i-j-1}\right]+\left(\begin{array}{l}
i+3 \\
i+2
\end{array}\right) e_{1}\left(u_{i}\right) .
\end{aligned}
$$

In small dimensions we have

$$
\begin{aligned}
& d\left(u_{1}\right)=0 \\
& d\left(u_{2}\right)=0 \\
& d\left(u_{3}\right)=0 \\
& d\left(u_{4}\right)=0 \\
& d\left(u_{5}\right)=e_{2}\left(u_{1}\right) \\
& d\left(u_{6}\right)=\left[u_{1}, u_{2}\right]+e_{1}\left(u_{2}\right)
\end{aligned}
$$




$$
d\left(u_{8}\right)=\left[u_{1}, u_{4}\right]+\left[u_{2}, u_{3}\right] .
$$

From these formulas it follows that the homology $H_{i}=H_{i}\left(\Omega^{3}\left(R P^{\infty} / R P^{3}\right)\right)$ in small dimensions $i$ has the following generators

$H_{1}: u_{1}$

$H_{2}: u_{1}^{2}, u_{2}$;

$H_{3}: u_{1}^{3}, u_{1} u_{2}, e_{1}\left(u_{1}\right), u_{3}$;

$H_{4}: u_{1}^{4}, u_{1}^{2} u_{2}, u_{1} e_{1}\left(u_{1}\right), u_{1} u_{3}, u_{2}^{2}, u_{4}$;

$H_{5}: u_{1}^{5}, u_{1}^{3} u_{2}, u_{1}^{2} u_{3}, u_{1}^{2} e_{1}\left(u_{1}\right), u_{1} u_{2}^{2}, u_{1} u_{4}, u_{2} u_{3}, u_{2} e_{1}\left(u_{1}\right), e_{1}\left(u_{2}\right)$;

$H_{6}: u_{1}^{6}, u_{1}^{4} u_{2}, u_{1}^{3} u_{3}, u_{1}^{3} e_{1}\left(u_{1}\right), u_{1}^{2} u_{2}^{2}, u_{1}^{2} u_{4}, u_{1} u_{2} u_{3}, u_{1} u_{2} e_{1}\left(u_{1}\right), u_{1} e_{1}\left(u_{2}\right)$, $u_{2} u_{4}, u_{2}^{3}, u_{3} e_{1}\left(u_{1}\right), u_{3}^{2}, e_{2}\left(u_{2}\right), e_{1}\left(u_{1}\right)^{2}$;

$H_{7}: u_{1}^{7}, u_{1}^{5} u_{2}, u_{1}^{4} u_{3}, u_{1}^{4} e_{1}\left(u_{1}\right), u_{1}^{3} u_{2}^{2}, u_{1}^{3} u_{4}, u_{1}^{2} u_{2} u_{3}, u_{1}^{2} u_{2} e_{1}\left(u_{1}\right), u_{1}^{2} e_{1}\left(u_{2}\right)$, $u_{1} u_{2} u_{4}, u_{1} u_{2}^{3}, u_{1} u_{3} e_{1}\left(u_{1}\right), u_{1} u_{3}^{2}, u_{1} e_{2}\left(u_{2}\right), u_{1} e_{1}\left(u_{1}\right)^{2}, u_{2}^{2} u_{3}, u_{2}^{2} e_{1}\left(u_{1}\right), u_{2} e_{1}\left(u_{2}\right)$, $u_{3} u_{4},\left[u_{2}, u_{3}\right], e_{1}\left(u_{3}\right), e_{1} e_{1}\left(u_{1}\right)$.

Consider also the homology $H_{*}\left(\Omega^{4}\left(R P^{\infty} / R P^{4}\right)\right)$. It is isomorphic to the homology of the differential $\mathcal{P}_{3}$-algebra $\mathcal{P}_{3 \varphi}\left\{u_{i} \mid i \geq 1\right\}$, where $u_{i}=s^{-4} e_{i+4}$ and the differential is determined by the formulas

$$
\begin{aligned}
& d_{\varphi}\left(u_{2 i+1}\right)=\sum_{j<i-1}\left[u_{j}, u_{2 i-j-3}\right]+\left(\begin{array}{l}
i+4 \\
i+1
\end{array}\right) e_{0}\left(u_{i}\right)+\left(\begin{array}{c}
i+3 \\
i+2
\end{array}\right) e_{2}\left(u_{i-1}\right) ; \\
& d_{\varphi}\left(u_{2 i+2}\right)=\sum_{j<i-1}\left[u_{j}, u_{2 i-j-2}\right]+\left(\begin{array}{l}
i+4 \\
i+2
\end{array}\right) e_{1}\left(u_{i}\right)+e_{3}\left(u_{i-1}\right) .
\end{aligned}
$$

In small dimensions we have

$$
\begin{aligned}
& d\left(u_{1}\right)=0 \\
& d\left(u_{2}\right)=0 \\
& d\left(u_{3}\right)=0 \\
& d\left(u_{4}\right)=0 \\
& d\left(u_{5}\right)=e_{2}\left(u_{1}\right) \\
& d\left(u_{6}\right)=e_{1}\left(u_{2}\right)+e_{3}\left(u_{1}\right) \\
& d\left(u_{7}\right)=\left[u_{1}, u_{2}\right]+u_{3} u_{3} \\
& d\left(u_{8}\right)=\left[u_{1}, u_{3}\right]+e_{1}\left(u_{3}\right)+e_{3}\left(u_{2}\right) .
\end{aligned}
$$

From these formulas it follows that the homology $H_{i}=H_{i}\left(\Omega^{4}\left(R P^{\infty} / R P^{4}\right)\right)$ in small dimensions $i$ has the following generators

$H_{1}: u_{1}$

$H_{2}: u_{1}^{2}, u_{2}$;

$H_{3}: u_{1}^{3}, u_{1} u_{2}, e_{1}\left(u_{1}\right), u_{3}$;

$H_{4}: u_{1}^{4}, u_{1}^{2} u_{2}, u_{1} e_{1}\left(u_{1}\right), u_{1} u_{3}, u_{2}^{2}, u_{4}$;

$H_{5}: u_{1}^{5}, u_{1}^{3} u_{2}, u_{1}^{2} u_{3}, u_{1}^{2} e_{1}\left(u_{1}\right), u_{1} u_{2}^{2}, u_{1} u_{4}, u_{2} u_{3}, u_{2} e_{1}\left(u_{1}\right), e_{1}\left(u_{2}\right)$;

$H_{6}: u_{1}^{6}, u_{1}^{4} u_{2}, u_{1}^{3} u_{3}, u_{1}^{3} e_{1}\left(u_{1}\right), u_{1}^{2} u_{2}^{2}, u_{1}^{2} u_{4}, u_{1} u_{2} u_{3}, u_{1} u_{2} e_{1}\left(u_{1}\right), u_{1} e_{1}\left(u_{2}\right)$, $u_{2}^{3}, u_{2} u_{4}, u_{3} e_{1}\left(u_{1}\right), u_{3}^{2}, e_{2}\left(u_{2}\right), e_{1}\left(u_{1}\right)^{2}$;

$H_{7}: u_{1}^{7}, u_{1}^{5} u_{2}, u_{1}^{4} u_{3}, u_{1}^{4} e_{1}\left(u_{1}\right), u_{1}^{3} u_{2}^{2}, u_{1}^{3} u_{4}, u_{1}^{2} u_{2} u_{3}, u_{1}^{2} u_{2} e_{1}\left(u_{1}\right), u_{1}^{2} e_{1}\left(u_{2}\right)$, $u_{1} u_{2}^{3}, u_{1} u_{2} u_{4}, u_{1} u_{3} e_{1}\left(u_{1}\right), u_{1} u_{3}^{2}, u_{1} e_{2}\left(u_{2}\right), u_{1} e_{1}\left(u_{1}\right)^{2}, u_{2}^{2} u_{3}, u_{2}^{2} e_{1}\left(u_{1}\right), u_{2} e_{1}\left(u_{2}\right)$, $u_{3} u_{4}, e_{1}\left(u_{3}\right), e_{3}\left(u_{2}\right), e_{1} e_{1}\left(u_{1}\right)$. 
Here we consider the problem of calculation the homology

$$
H_{*}\left(\Omega^{m}\left(C P^{\infty} / C P^{n}\right)\right), \quad m \leq 2 n+1,
$$

of iterated loop spaces of the space $C P^{\infty} / C P^{n}$. We begin with $Z / 2$ coefficients.

Denote the $2 i$-dimensional generator of $H_{*}\left(C P^{\infty}\right)$ as $c_{i}$. A coalgebra structure on this generators is determined by the formula

$$
\nabla\left(c_{i}\right)=\sum_{j} c_{j} \otimes c_{i-j} .
$$

An action of the Steenrod algebra is determined by the formula

$$
S q^{2 j}\left(c_{i}\right)=\left(\begin{array}{c}
i-j \\
j
\end{array}\right) c_{i-j}
$$

As above, since $H_{*}\left(C P^{\infty}\right)$ is the free $\bar{E}_{*^{-}}$coalgebra there are no higher $\bar{E}_{*^{-}}$ operations. The homology $H_{*}\left(C P^{n}\right)$ is the $\bar{E}_{*}$-subcoalgebra of $H_{*}\left(C P^{\infty}\right)$ and hence the $\bar{E}_{*}$-coalgebra structure on $H_{*}\left(C P^{\infty}\right)$ induces the $\bar{E}_{*}$-coalgebra structure on

$$
H_{*}\left(C P^{\infty}\right) / H_{*}\left(C P^{n}\right) \cong H_{*}\left(C P^{\infty} / C P^{n}\right)
$$

Therefore on the homology $H_{*}\left(C P^{\infty} / C P^{n}\right)$ there are no higher $\bar{E}_{*}$-operations. So the spectral sequence of the homology of the iterated loop spaces of $C P^{\infty} / C P^{n}$ has no higher differentials and we have

Theorem 7. The homology $H_{*}\left(\Omega^{m}\left(C P^{\infty} / C P^{n}\right)\right), m \leq 2 n+1$ (over $Z / 2$ ) is isomorphic to the homology of the $\mathcal{P}_{m-1}$-algebra $\mathcal{P}_{m-1 \varphi} S^{-m} H_{*}\left(C P^{\infty} / C P^{n}\right)$, where the differential $d_{\varphi}$ on the generators $s^{-m} c_{i}$ is defined by the formula

$$
d_{\varphi}\left(s^{-m} c_{i}\right)=\sum_{\substack{j \\
2 j<i}}\left[s^{-m} c_{j}, s^{-m} c_{i-j}\right]+\sum_{\substack{j \\
2 j \leq i}}\left(\begin{array}{c}
i-j \\
j
\end{array}\right) e_{2(2 j-i)+m-1}\left(s^{-m} c_{i-j}\right) .
$$

Note that in the case $m=1$ we have the isomorphisms

$$
\mathcal{P}_{0} S^{-1} H_{*}\left(C P^{\infty} / C P^{n}\right) \cong T_{s} L S^{-1} H_{*}\left(C P^{\infty} / C P^{n}\right) \cong T S^{-1} H_{*}\left(C P^{\infty} / C P^{n}\right),
$$

and the differential in the tensor algebra $T S^{-1} H_{*}\left(C P^{\infty} / C P^{n}\right)$ on the generators $v_{i}=s^{-1} c_{i+1}$ of dimensions $2 i+1$ has a very simple form:

$$
d_{\varphi}\left(v_{i}\right)=\sum_{j} v_{j} \otimes v_{i-j-1}
$$

From here it follows that the homology $H_{*}\left(\Omega\left(C P^{\infty} / C P^{n}\right)\right)($ over $Z / 2)$ is isomorphic to the algebra generated by the elements $v_{i}, n \leq i \leq 2 n$, and relations

$$
\begin{gathered}
v_{n} \cdot v_{n}=0 \\
v_{n} \cdot v_{n+1}+v_{n+1} \cdot v_{n}=0
\end{gathered}
$$


So as a graded module the homology $H_{*}\left(\Omega\left(C P^{\infty} / C P^{n}\right)\right)$ is generated by the noncommutative products $v_{n_{1}} \cdot \ldots \cdot v_{n_{k}}$ with $n \leq n_{1} \leq 2 n, n<n_{2}, \ldots, n_{k} \leq 2 n$.

Consider the homology $H_{*}\left(\Omega^{2}\left(C P^{\infty} / C P^{n}\right)\right)$. It is isomorphic to the homology of the differential $\mathcal{P}_{1}$-algebra $\mathcal{P}_{1 \varphi}\left\{v_{i} \mid i \geq n\right\}$, where $v_{i}=s^{-2} c_{i+1}, \operatorname{dim}\left(v_{i}\right)=2 i$ and the differential is determined by the formulas

$$
\begin{aligned}
d_{\varphi}\left(v_{2 i}\right) & =\sum_{j<i}\left[v_{j}, v_{2 i-j-1}\right] \\
d_{\varphi}\left(v_{2 i+1}\right) & =\sum_{j<i}\left[v_{j}, v_{2 i-j}\right]+e_{1}\left(v_{i}\right)
\end{aligned}
$$

From these formulas it follows that the homology $H_{*}\left(\Omega^{2}\left(C P^{\infty} / C P^{n}\right)\right)$ is the $\mathcal{P}_{1}$-algebra generated by the elements $v_{n}, \ldots, v_{2 n}$ and relations

$$
\begin{gathered}
e_{1}\left(v_{n}\right)=0 \\
{\left[v_{n}, v_{n+1}\right]=0 ;} \\
{\left[v_{n}, v_{n+2}\right]+e_{1}\left(v_{n+1}\right)=0} \\
\cdots \\
{\left[v_{n}, v_{2 n}\right]+\left[v_{n+1}, v_{2 n-1}\right]+\cdots=0 .}
\end{gathered}
$$

Consider the homology $H_{*}\left(\Omega^{4}\left(C P^{\infty} / C P^{2}\right)\right)$. It is isomorphic to the homology of the differential $\mathcal{P}_{3}$-algebra $\mathcal{P}_{3 \varphi}\left\{v_{i} \mid i \geq 1\right\}$, where $v_{i}=s^{-4} c_{i+2}, \operatorname{dim}\left(v_{i}\right)=2 i$ and the differential is determined by the formulas

$$
\begin{aligned}
& d_{\varphi}\left(v_{2 i+1}\right)=\sum_{j<i}\left[v_{j}, v_{2 i-j-1}\right]+\left(\begin{array}{c}
i+2 \\
i+1
\end{array}\right) e_{1}\left(v_{i}\right) \\
& d_{\varphi}\left(v_{2 i+2}\right)=\sum_{j<i}\left[v_{j}, v_{2 i-j}\right]+e_{3}\left(v_{i}\right) .
\end{aligned}
$$

In small dimensions we have

$$
\begin{aligned}
& d\left(v_{1}\right)=0 ; \\
& d\left(v_{2}\right)=0 \\
& d\left(v_{3}\right)=e_{1}\left(v_{1}\right) \\
& d\left(v_{4}\right)=e_{3}\left(v_{1}\right) \\
& d\left(v_{5}\right)=\left[v_{1}, v_{2}\right] \\
& d\left(v_{6}\right)=\left[v_{1}, v_{3}\right]+e_{3}\left(v_{2}\right) ; \\
& d\left(v_{7}\right)=\left[v_{1}, v_{4}\right]+\left[v_{2}, v_{3}\right]+e_{1}\left(v_{3}\right) ;
\end{aligned}
$$

From these formulas it follows that the homology $H_{i}=H_{i}\left(\Omega^{4}\left(C P^{\infty} / C P^{2}\right)\right)$ in small dimensions $i$ has the following generators

$$
\begin{aligned}
& H_{1}=0 ; \\
& H_{2}: \quad v_{1} ; \\
& H_{3}=0 ; \\
& H_{4}: \quad v_{1}^{2}, v_{2} ; \\
& H_{5}=0 ; \\
& H_{6}: \quad v_{1}^{3}, v_{1} v_{2}, e_{2}\left(v_{1}\right) ;
\end{aligned}
$$


$H_{8}: \quad v_{1}^{4}, v_{1}^{2} v_{2}, v_{1} e_{2}\left(v_{1}\right), v_{2}^{2}$

$H_{9}: \quad e_{1}\left(v_{2}\right)$

$H_{10}: v_{1}^{5}, v_{1}^{3} v_{2}, v_{1}^{2} e_{2}\left(v_{1}\right), v_{1} v_{2}^{2}, v_{2} e_{2}\left(v_{1}\right), e_{2}\left(v_{2}\right)$;

$H_{11}: v_{1} e_{1}\left(v_{2}\right), e_{3}\left(v_{2}\right)$

$H_{12}: v_{1}^{6}, v_{1}^{4} v_{2}, v_{1}^{3} e_{2}\left(v_{1}\right), v_{1}^{2} v_{2}^{2}, v_{1} v_{2} e_{2}\left(v_{1}\right), v_{1} e_{2}\left(v_{2}\right)$;

$H_{13}: v_{1}^{2} e_{1}\left(v_{2}\right), v_{1} e_{3}\left(v_{2}\right), e_{1}\left(v_{3}\right),\left[v_{2}, v_{3}\right]$.

Consider now $Z / p$ coefficients, $p>2$. The coalgebra structure on the generators is determined by the formula

$$
\nabla\left(c_{i}\right)=\sum_{j} c_{j} \otimes c_{i-j} .
$$

The action of the mod-p Steenrod algebra is determined by the formula

$$
P^{j}\left(c_{i}\right)=\left(\begin{array}{c}
i-(p-1) j \\
j
\end{array}\right) c_{i-(p-1) j} .
$$

Since $H_{*}\left(C P^{\infty}\right)$ is the free $\bar{E}_{*}$-coalgebra, there are no higher $\bar{E}_{*}$-operations.

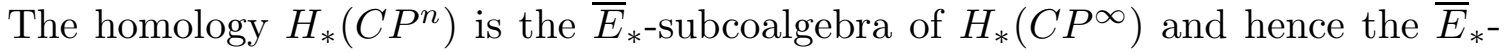
coalgebra structure on $H_{*}\left(C P^{\infty}\right)$ induces the $\bar{E}_{*}$-coalgebra structure on

$$
H_{*}\left(C P^{\infty}\right) / H_{*}\left(C P^{n}\right) \cong H_{*}\left(C P^{\infty} / C P^{n}\right) .
$$

Therefore on the homology $H_{*}\left(C P^{\infty} / C P^{n}\right)$ there are no higher $\bar{E}_{*}$-operations. So the spectral sequence of the homology of the iterated loop spaces of $C P^{\infty} / C P^{n}$ has no higher differentials and we have

Theorem 8. The homology $H_{*}\left(\Omega^{m}\left(C P^{\infty} / C P^{n}\right)\right), m \leq 2 n+1$ (over $Z / p$ ) is isomorphic to the homology of the $\mathcal{P}_{m-1}$-algebra $\mathcal{P}_{m-1 \varphi} S^{-m} H_{*}\left(C P^{\infty} / C P^{n}\right)$, where the differential $d_{\varphi}$ on the generators $s^{-m} e_{i}$ is defined by the formula

$$
\begin{aligned}
d_{\varphi}\left(s^{-m} c_{i}\right) & =\sum_{\substack{j \\
2 j \leq i}}\left[s^{-m} c_{j}, s^{-m} c_{i-j}\right]+ \\
& +\sum_{\substack{j \\
p j<i}}\left(\begin{array}{c}
i-(p-1) j \\
j
\end{array}\right) \beta e_{2(p j-i)+m}\left(s^{-m} c_{i-(p-1) j}\right) .
\end{aligned}
$$

Note that in the case $m=1$ we have the isomorphisms

$$
\mathcal{P}_{0} S^{-1} H_{*}\left(C P^{\infty} / C P^{n}\right) \cong T_{s} L S^{-1} H_{*}\left(C P^{\infty} / C P^{n}\right) \cong T S^{-1} H_{*}\left(C P^{\infty} / C P^{n}\right),
$$

and the differential in the tensor algebra $T S^{-1} H_{*}\left(C P^{\infty} / C P^{n}\right)$ has very simple form

$$
d_{\varphi}\left(s^{-1} c_{i}\right)=\sum_{j} s^{-1} c_{j} \otimes s^{-1} c_{i-j} .
$$

From here it follows that the homology $H_{*}\left(\Omega\left(C P^{\infty} / C P^{n}\right)\right)$ (over $\left.Z / p\right)$ is isomorphic to the algebra generated by the elements $v_{i}=s^{-1} c_{i+1}, n \leq i \leq 2 n$, of dimensions $2 i+1$ and relations

$$
\begin{gathered}
v_{n} \cdot v_{n}=0 \\
v_{n} \cdot v_{n+1}+v_{n+1} \cdot v_{n}=0
\end{gathered}
$$


So as a graded module the homology $H_{*}\left(\Omega\left(C P^{\infty} / C P^{n}\right)\right)$ is generated by the noncommutative products $v_{n_{1}} \cdot \ldots \cdot v_{n_{k}}$ with $n \leq n_{1} \leq 2 n, n<n_{2}, \ldots, n_{k} \leq 2 n$.

Consider the homology $H_{*}\left(\Omega^{2}\left(C P^{\infty} / C P^{n}\right)\right)$. It is isomorphic to the homology of the differential $\mathcal{P}_{1}$-algebra $\mathcal{P}_{1 \varphi}\left\{v_{i} \mid i \geq 1\right\}$, where $v_{i}=s^{-2} c_{i+1}, i \geq n$ and the differential is determined by the formulas

$$
d_{\varphi}\left(v_{i}\right)=\sum_{2 j<i}\left[v_{j}, v_{i-j-1}\right]
$$

From these formulas it follows that the homology $H_{i}=H_{i}\left(\Omega^{2}\left(C P^{\infty} / C P^{n}\right)\right)$ is the $\mathcal{P}_{1}$-algebra generated by the elements $v_{n}, \ldots, v_{2 n}$ and relations

$$
\begin{gathered}
{\left[v_{n}, v_{n}\right]=0} \\
{\left[v_{n}, v_{n+1}\right]=0 ;} \\
{\left[v_{n}, v_{n+2}\right]+\left[v_{n+1}, v_{n+1}\right]=0} \\
\cdots \\
{\left[v_{n}, v_{2 n}\right]+\left[v_{n+1}, v_{2 n-1}\right]+\cdots=0 .}
\end{gathered}
$$

Consider the homology $H_{*}\left(\Omega^{4}\left(C P^{\infty} / C P^{2}\right)\right)$. It is isomorphic to the homology of the differential $\mathcal{P}_{3}$-algebra $\mathcal{P}_{3 \varphi}\left\{v_{i} \mid i \geq 1\right\}$, where $v_{i}=s^{-4} c_{i+2}, \operatorname{dim}\left(v_{i}\right)=2 i$ and the differential is determined by the formulas

$$
\begin{aligned}
d_{\varphi}\left(v_{p i-1}\right) & =\sum_{2 j<p i-2}\left[v_{j}, v_{p i-j-3}\right]+\left(\begin{array}{c}
i+1 \\
i
\end{array}\right) \beta e_{2}\left(v_{i-1}\right) ; \\
d_{\varphi}\left(v_{k}\right) & =\sum_{2 j<k-1}\left[v_{j}, v_{k-j-2}\right], k \neq p i-1 .
\end{aligned}
$$

In small dimensions, we have

$d\left(v_{1}\right)=0$

$d\left(v_{2}\right)=0$

$d\left(v_{3}\right)=0$

$d\left(v_{4}\right)=\left[v_{1}, v_{1}\right]$

$d\left(v_{5}\right)=\left[v_{1}, v_{2}\right]$

$d\left(v_{6}\right)=\left[v_{1}, v_{3}\right]+\left[v_{2}, v_{2}\right]$

$\cdots$

$d\left(v_{2 p-1}\right)=\left[v_{1}, v_{2 p-4}\right]+\cdots+\left[v_{p-2}, v_{p-1}\right]+3 \beta e_{2}\left(v_{1}\right)$

...

From these formulas it follows that the homology $H_{i}=H_{i}\left(\Omega^{4}\left(C P^{\infty} / C P^{2}\right)\right)$ with, for example, $Z / 3$-coefficients, in small dimensions $i$ has the following generators

$H_{1}=0$

$H_{2}: v_{1}$

$H_{3}=0$

$H_{4}: v_{1}^{2}, v_{2}$

$H_{5}=0$;

$H_{6}: v_{1}^{3}, v_{1} v_{2}, v_{3}$; 


$$
\begin{array}{ll}
H_{8}: & v_{1}^{4}, v_{1}^{2} v_{2}, v_{1} v_{3}, v_{2}^{2} \\
H_{9}: & \beta e_{2}\left(v_{1}\right) \\
H_{10}: & v_{1}^{5}, v_{1}^{3} v_{2}, v_{1}^{2} v_{3}, v_{1} v_{2}^{2}, v_{2} v_{3}, e_{2}\left(v_{1}\right) \\
H_{11}: & v_{1} \beta e_{2}\left(v_{1}\right),\left[v_{2}, v_{2}\right] .
\end{array}
$$

\section{APPENDIX}

\section{F. Sergeraert}

The general ideas of the paper [11] led Julio Rubio and myself to a simple method solving the computability problem for the homology groups of iterated loop spaces, when the initial space is sufficiently reduced. The main ingredient is functional Programming, allowing us to constructively apply three particular cases of the basic homological perturbation lemma in situations involving highly infinite simplicial sets; see [23].

This is not only a theoretical result. The algorithm, the existence of which has been so proved, has been concretely written in Common Lisp with significant results. The first version of our program, named EAT (Effective Algebraic Topology), is Web-reachable [24] with a rich documentation (250pp.). The most recent version of this program named Kenzo had just been finished when this appendix was written (January 1999). See [25] for a few explanations and a small demonstration file. A public version of this program, with a reasonably complete user guide, will be soon distributed at the same Web-address.

Let us give a typical example of the use of Kenzo, related to the main subject of this paper. What about the first $\mathbb{Z}$-homology groups of the iterated loop spaces $\Omega_{n}^{p}=\Omega^{p}\left(R P^{\infty} / R P^{n}\right)$, if $p \leq n$ ? The situation is difficult: the projective space is not a suspension and the classical results about the homology groups of spaces of the form $\Omega^{n} S^{n} X$ cannot be applied; see [18] for a survey of this subject. The only general solution known at this time to determine the groups $H_{n}\left(\Omega_{n}^{p} ; \mathbb{Z}_{2}\right)$, due to Vladimir Smirnov, is the subject of the main part of this paper. We explain here how the results of Smirnov can be verified with the Kenzo program for the first homology groups.

Let us consider for example the computation of $H_{5}\left(\Omega^{2}\left(R P^{\infty} / R P^{2}\right)\right)$. In an interactive Lisp session where the Kenzo program has been loaded, you can execute:

USER(1): (setf trunc3-proj-space ( $r$-proj-space 3)) ==>

[K1 Simplicial-Set]

This Lisp dialog must be understood as follows. The string USER(1): is the Lisp prompt; Lisp is waiting for the next expression you want to evaluate. In this case the instruction is :

(setf trunc3-proj-space ( $r$-proj-space 3 ))

which means you want to assign to the symbol trunc3-proj-space the result of the evaluation of ( $r$-proj-space 3 ). The last evaluation constructs in the Lisp environment our version of the truncated projective space $R P^{\infty} / R P^{2}$, and the resulting object is assigned to the symbol; furthermore a simple external form of the assigned object is displayed; this form can be read: this is the Kenzo object \#1 
$\mathrm{K} 1$, which is a simplicial set. Of course the internal form is much more complicated: the internal object is the looked-for space, or more precisely codes this space.

But we want to consider the second loop-space of the truncated projective space. The Kenzo program constructs it in this way:

USER(2): (setf omega2-trunc3-proj-space (loop-space trunc3-proj-space 2)) ==>

[K22 Simplicial-Group]

The result, the Kenzo object \# 22, is a simplicial group, namely the Kan model of the second loop space [21], a highly infinite object.

The homology groups of an object such as a chain complex, a simplicial set, are computed by the Kenzo function homology. For example if you want to verify that the truncated projective space has the right homology in dimension 5:

USER(3): (homology trunc3-proj-space 5) ==>

Computing boundary-matrix in dimension 5 .

Rank of the source-module : 1 .

Computing boundary-matrix in dimension 6 .

Rank of the source-module : 1 .

Homology in dimension 5 :

Component $\mathrm{Z} / 2 \mathrm{Z}$

---done---

The homology group is $Z_{2}$. The homology group $H_{5}\left(\Omega^{2}\left(R P^{\infty} / R P^{2}\right)\right)$ is obtained in the same way:

USER (4): (homology omega2-trunc3-proj-space 5) ==>

Computing boundary-matrix in dimension 5 .

Rank of the source-module : 29.

Computing boundary-matrix in dimension 6 .

Rank of the source-module : 70 .

Homology in dimension 5 :

Component $\mathrm{Z} / 2 \mathrm{Z}$

Component $\mathrm{Z} / 2 \mathrm{Z}$

---done---

So that $H_{5}\left(\Omega^{2}\left(R P^{\infty} / R P^{2}\right)\right)=Z_{2}^{2}$. As you see, the program determines a Hirsch $Z$-complex for our loop space, with 29 generators in dimension 5 and 70 generators in dimension 6 . Note the program gives you the $Z$-homology, not the $Z_{2}$-homology, as in Smirnov's text. The complexity is very high: the highest group computed by Kenzo for this second loop space is $H_{7}=Z_{2}^{2} \oplus Z_{8}$; one day of CPU time has been needed on a powerful Linux PC to obtain it.

The topologists frequently ask for some homology groups determined by the Kenzo program that are not reachable by human computers. The first example of this sort was given by the previous version of the Kenzo program, the EAT program [24]. We repeat it here, because it is very simple and ten years after its first computation, we have not yet found a topologist knowing how to compute this group by hand. Please try to do it!

The construction is the following. The homotopy group $\pi_{2} \Omega S^{3}$ is $Z$, so that attaching a 3-cell to $\Omega S^{3}$ by a map of degree 2 makes sense. Let $D \Omega S^{3}$ the space so obtained. Question: what about the homology groups of $\Omega D \Omega S^{3}$ ? Let us show how the first homology groups of this strange loop space are computed by the Kenzo program. Firstly the loop space $\Omega S^{3}$ is constructed as before: 
[K262 Simplicial-Set]

USER(6): (setf os3 (loop-space s3)) ==>

[K267 Simplicial-Group]

How to attach a 3-cell? This 3-cell will be a 3-simplex and, in order to attach it, we must describe what 2-simplices of $\Omega S^{3}$ are its faces. The list of faces is defined and used as follows:

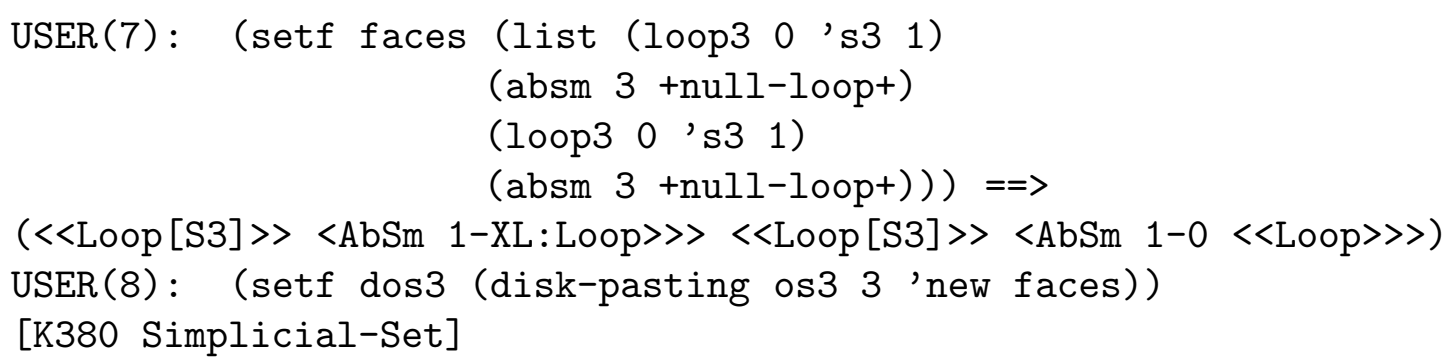

The disk-pasting function atttaches a 3-cell of "name" new to os3, using the face list faces; this list is:

0 ) The "fundamental" simplex of $\Omega S^{3}$;

1 ) The second face is collapsed on the base point;

2 ) The same as 0);

3 ) The same as 2);

so that, taking account of the usual sign rules, the attaching map has degree 2 . The space so constructed is assigned to the symbol dos3. The second homology group of this space is verified:

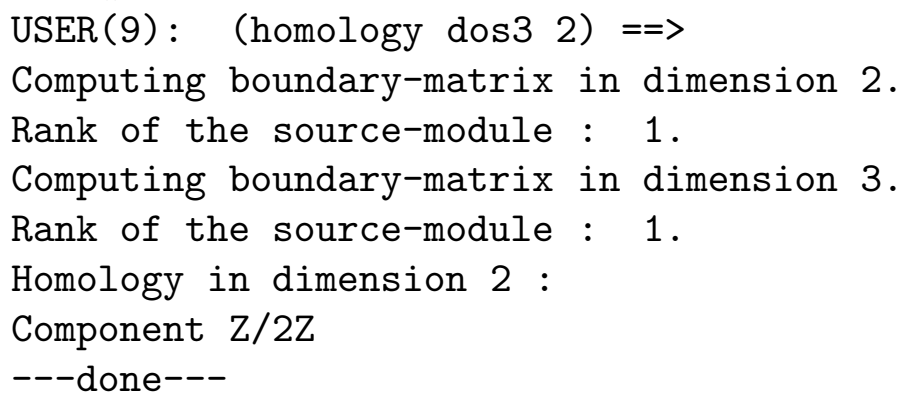

Finally we construct the loop space $\Omega D \Omega S^{3}$ and compute the sixth homology group:

USER(10): (setf odos3 (loop-space dos3)) ==>

[K398 Simplicial-Group]

USER(11): (homology odos3 6) ==>

Computing boundary-matrix in dimension 6 .

Rank of the source-module : 26.

Computing boundary-matrix in dimension 7 .

Rank of the source-module : 50 .

Homology in dimension 6 :

Component Z/6Z

Component $\mathrm{Z} / 2 \mathrm{Z}$

Component $\mathrm{Z} / 2 \mathrm{Z}$

Component $\mathrm{Z} / 2 \mathrm{Z}$

Component $\mathrm{Z} / 2 \mathrm{Z}$

Component $\mathrm{Z} / 2 \mathrm{Z}$

Component $\mathrm{Z} / 2 \mathrm{Z}$

Component Z/2Z 
Component $\mathrm{Z} / 2 \mathrm{Z}$

Component $\mathrm{Z} / 2 \mathrm{Z}$

Component $\mathrm{Z} / 2 \mathrm{Z}$

Component $\mathrm{Z} / 2 \mathrm{Z}$

---done---

$H_{6}\left(\Omega D \Omega S^{3}\right)=Z_{2}^{12} \oplus Z_{6}$. The Kenzo program has determined these groups up to dimension 9 in a few days.

\section{REFERENCES}

1. J.F.Adams. On the cobar construction. Proc. Nat. Acad. Sci. 42(1956), 409-412.

2. H.J.Baues. The double bar and cobar constructions. Comp. Math. 43(1981), $331-341$.

3. J.P.May. The Geometry of Iterated Loop Spaces. Lect. Notes in Math. 1972 v. 271 .

4. F.R.Cohen, T.J.Lada, J.P.May. The Homology of Iterated Loop Spaces. Lect. Notes in Math. 1976, v. 533.

5. E.Dyer, R.Lashof. Homology of iterated loop spaces. Amer. J. Math. 84(1962), 35-88.

6. R.J.Milgram. Iterated loop spaces. Ann. of Math. 84(1966), N 3, 386-403.

7. V.A.Smirnov. On the cochain complex of topological spaces. Mat. Sb. (Russia), 115(1981), 146-158.

8. V.A.Smirnov. Homotopy theory of coalgebras. Izv. Ac. Nauk (Russia), 49(1985), 1302-1321.

9. V.A.Smirnov. On the chain complex of an iterated loop space. Izv. Ac. Nauk (Russia), 53(1989), 1108-1119.

10. F.Cohen, R.Levi. On the homotopy type of infinite stunted projective spaces. To appear in BCAT 1998.

11. F.Sergeraert. The computability Problem in Algebraic Topology. Advances in Math. 104(1994), N 1, 1-29.

12. A.Vinogradov, M.Vinogradov. On multiple generalizations of Lie algebras and Poisson manifolds. Secondary calculus and cohomological physics (Moscow, 1997). Amer. Math. Soc. Providence, RI, 1998, p. 273-287.

13. J.M.Boardman and R.M.Vogt. Homotopy invariant algebraic structures on topological spaces. Lect. Notes in Math. 347, 1973.

14. S.Araki, T.Kudo. Topology of $H_{n}$-spaces and $H$-squaring operations. Mem. Fac. Sci. Kyusyu Univ., Ser. A, 10(1956), N 2, 85-120.

15. F.P.Peterson. Functional cohomology operations. Trans. Amer. Math. Soc. 86(1957), p.187-197.

16. V.A.Smirnov. Functional homology operations and weak homotopy type. Mat. Zametki (Russia), 45(1989), N 5, p.76-86.

17. R. Brown. The twisted Eilenberg-Zilber theorem. Celebrazioni Arch. Secolo XX, Simp. Top., 1967, 34-37.

18. G. Carlsson, R. J. Milgram. Stable homotopy and iterated loop spaces. In [20], 505-583.

19. V.K.A.M. Gugenheim. On a perturbation theory for the homology of the loop 
20. Handbook of Algebraic Topology (Edited by I.M. James). North-Holland, 1995.

21. D. M. Kan. A combinatorial definition of homotopy groups. Annals of Mathematics. 67(1958), 282-312.

22. J. Rubio, F. Sergeraert. A program computing the homology groups of loop spaces. SIGSAM Bulletin, 25(1991), 20-24.

23. J. Rubio, F. Sergeraert. Constructive Algebraic Topology. In preparation, see ftp:// www-fourier.ujf-grenoble.fr/ sergerar.

24. J. Rubio, F. Sergeraert, Y. Siret. The EAT program. ftp://www-fourier.ujfgrenoble.fr/ ftp/EAT.

25. F. Sergeraert. The Kenzo program. http://www-fourier.ujf-grenoble. fr/ sergerar/Kenzo/ 